\title{
ORGANIZATION OF OCCURRENCE-RELATED BIODIVERSITY RESOURCES BASED ON THE PROCESS OF THEIR CREATION AND THE ROLE OF INDIVIDUAL ORGANISMS AS RESOURCE RELATIONSHIP NODES
}

\author{
STEVEN J. BASKAUF \\ Department of Biological Sciences \\ Vanderbilt University, Nashville,TN, USA, steve.baskauf@vanderbilt.edu
}

\begin{abstract}
Kinds of occurrences (evidence of particular living organisms) can be grouped by common data and metadata characteristics that are determined by the way that the occurrence represents the organism. The creation of occurrence resources follows a pattern which can be used as the basis for organizing both the metadata associated with those resources and the relationships among the resources. The central feature of this organizational system is a resource representing the individual organism. This resource serves as a node which connects the organism's occurrences and any determinations of the organism's taxonomic identity. I specify a relatively small number of predicates which can define the important relationships among these resources and suggest which metadata properties should logically be associated with each kind of resource.
\end{abstract}

Key words. - GUID, individual, metadata, occurrence, RDF

Traditionally, biodiversity informatics has involved the discovery and compilation of specimen data, and the application of those data to determine where organisms live. With the development of computers and the Internet, the capability now exists to aggregate and disseminate biodiversity data globally as well as to broaden the scope beyond that of establishing species ranges (Kelling, 2008). Currently there is a coordinated effort to create the data infrastructure, standardize metadata terms (Darwin Core Task Group, 2009; Jones et al. 2009), and develop persistent, actionable identifiers (globally unique identifiers or GUIDs; Cryer et al. 2009, Richards 2009) in order to facilitate the aggregation of not only specimen metadata, but metadata derived from observations and digital representations of live organisms such as images.

An important aspect of the development of this global network is creating the capacity for users (and the computer applications that assist them) to find other resources that are related to a resource which is at hand, i.e. "linked data" ${ }^{1}$. Implicit in the concept of persistent identifiers is the understanding such identifiers are also resolvable, meaning that knowledge of the identifier also provides a means to access the information (data and metadata) for the resource to

\footnotetext{
${ }^{1}$ http://www4.wiwiss.fu-berlin.de/bizer/pub/LinkedDataTutorial/
}

which the identifier refers. An important part of this information is the relationship of the resource to related resources, but using these links to related resources is only possible if the relationships are specified in a consistent way that can be understood by all applications that wish to use them.

In this paper, I suggest a system of organizing biodiversity resources around individual organism records. These records serve as nodes for grouping related occurrence resources and their taxonomic determinations. Because this system is based on the way occurrence resources are created, it connects the resources in a logically consistent way and associates metadata properties with resources in an efficient manner.

\section{Definitions}

In this paper, these terms are used according to their standard meaning as a defined in the citations or footnotes. The exceptions are abstract, conceptual, and defined abstract resources which for the purposes of this paper have been assigned specific meanings as defined below.

resource - A physical, digital or conceptual entity which can be identified by a Uniform Resource Identifier (URI) (Berners-Lee et al. 2005).

information resource - A resource for which all essential characteristics can be transmitted in a 
message (Jacobs and Walsh 2004), i.e. a digital resource. Examples: text and digital images.

data - The content of the message that provides the representation of the information resource.

non-information resource - A resource that cannot be transmitted electronically. Technically, a resource is defined as a non-information resource when an HTTP GET request for the resource does not result in a 2xx "Success" response, i.e. no data are returned (W3C Technical Architecture Group 2005). Examples: persons, specimens, observations, and taxonomic concepts.

metadata - Data about data ${ }^{2}$. All resources can have metadata that describe their properties.

physical resource - A non-information resource that is a material thing ${ }^{3}$. Examples: living organisms, specimens, and $35 \mathrm{~mm}$ slide images.

abstract resource - A non-information resource that does not represent a particular material thing. Examples: observations, protein structures, state boundaries, and concepts.

conceptual resource - An abstract resource that is subject to varying interpretation. Examples: relationships, taxonomic concepts, and properties.

defined abstract resource - A resource which represents a defined circumstance or abstract object, and which therefore is not subject to interpretation. Examples: observations, determinations, and mathematical concepts.

HTTP URI - An identifier beginning with "http://" used to name resources on the World Wide Web ("the Web"). An HTTP URI may or may not refer to a retrievable resource on the Web. HTTP URIs uniquely identify a single resource.

node - A resource in a data structure which is linked to other resources by references to them. In diagrams in this paper, nodes are represented by geometric shapes and the links between them are represented by lines or arrows.

\section{RESOURCES IN A BIODIVERSITY CONTEXT \\ Characteristics of occurrences resources}

There are a number of categories of resources that are of interest to the biodiversity community and all of the types of resources defined above are represented in these resource categories. The primary focus of this paper is on resources that are

\footnotetext{
${ }^{2}$ http://dublincore.org/metadata-basics/

3 http://purl.org/dc/terms/PhysicalResource
}

instances of the Darwin Core class Occurrence, which is defined as "the category of information pertaining to evidence of an occurrence in nature, in a collection, or in a dataset (specimen, observation, etc.)" (Darwin Core Task Group 2009). Although the name "Occurrence" suggests that such resources serve the purpose of documenting that an individual or population of individuals occurred at a particular time and place, categorizing a resource as an occurrence does not imply fitness for that use or any other particular use. An occurrence resource could also be used to document character states, to serve as a type specimen, to be used as a logo, or any combination of these or other uses.

It should be clarified that the scope of this paper is restricted to Occurrences as a subset of a broader category of biodiversity resources defined by Kelling (2008) as "observational data". The scope of the paper includes observational data that document the presence of a single individual at a given point in time, but does not include observational data that are place-based (e.g. species checklists and measures of abundance; Kelling 2008). For the purposes of this paper, an occurrence is assumed to document a single individual. However, most of what is discussed here could also apply to small populations of individuals that are of the same species.

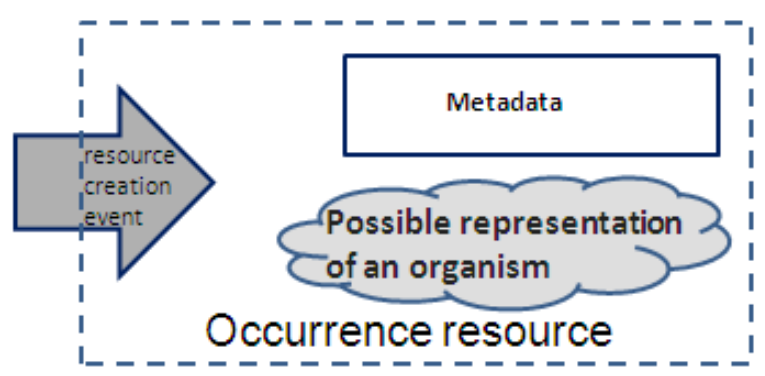

Fig. 1. Components of a generic occurrence resource

What occurrence resources have in common is that they assert that an organism occurred somewhere at some time. In this paper, I will describe the process by which occurrence resources come into existence and how this process can help us organize our thinking about the relationship between resources and the properties that should be associated with particular types of resources. Determining the associations and properties that are appropriate for particular 


\section{BASKAUF - ORGANIZATION OF OCCURRENCE-RELATED BIODIVERSITY RESOURCES}

resource types is critical for the successful implementation of an actionable GUID system.

In this paper I will distinguish between several aspects of occurrence resources. Each occurrence resource is the result of an event ${ }^{4}$ which results in the creation of the resource. During the event, a representation of the organism may be created. The resource and the possible representation with which the resource may be associated have properties that comprise the metadata for that resource. Thus the occurrence resource may have three aspects: a resource creation event, a representation of the organism, and metadata (Fig. $1)$.

\section{OCCURRENCE RESOURCE CREATION EVENTS}

In a resource creation event, the occurrence resource must be created from another resource. This follows from the fact that an occurrence resource is evidence of the existence of an organism. A resource such as a generic illustration of a bird which is not created from another particular resource is not an occurrence because it does not represent a particular organism. The resource creation event could itself be considered a separate resource with its own persistent identifier. However, because of the oneto-one relationship between the creation event and the created resource, it is more straightforward to simply consider the metadata for the event (i.e. time and location information) as a part of the metadata for the occurrence resource itself.

When I say that a resource was "created" I mean that the resource and any associated representation was caused to become a separate entity by whatever means is associated with that particular type of resource. For example, preserved specimens are collected, living specimens are planted or moved from their natural environment, digital images are photographed, DNA sequences are sequenced, etc. This is not saying that the creator necessarily caused the representation itself to come into existence. For example, a tree twig was actually "created" as a physical object by the tree, not the person who

${ }^{4}$ http://purl.org/dc/dcmitype/Event Note that the use of "occurrence" in the definition of Event is in the sense of "something happening" rather than the specific use of Occurrence sensu Darwin Core. collects it. However, the specimen that has the twig as its representation of the tree was created by the collector. Thus in this sense it is appropriate for all occurrence resources to use the Dublin Core (Dublin Core Metadata Initiative, $\mathrm{DCMI}^{5}$ ) term dcterms:creator ${ }^{6}$ ("an entity primarily responsible for making the resource") ${ }^{7}$ to indicate the person (or institution) who caused the resource to come into existence.

It is also important here to make the distinction between the event that results in the creation of the occurrence resource and the occurrence resource itself. For some resources, this distinction is obvious. For example, when a specimen is collected, there is an event (a segment of a collecting trip) that takes place at a particular time and place and the result of this event is a resource that we call a specimen. When an image of an organism is recorded, an event (a "photo shoot") that takes place at a particular time and place results in a resource we call a StillImage. Unfortunately this distinction is blurred in the case of an observation because we use the word "observation" to mean at least two different kinds of things. Webster's New World Dictionary provides two definitions of "observation" as "4 a) the act or practice of noting and recording facts and events, as for some scientific study b) the data so noted and recorded" (Neufeldt and Guralnik 1994). The first definition (a) represents an observation as an event (as in "we conducted an observation") and the second (b) represents an observation as the created resource (as in "our observations were written in a notebook"). Observation occurrences are resources of the second type. They have resource creation events, but they are not themselves events. (It is sometimes suggested that observation occurrences should be classed in the Dublin Core class Event, but they are no more events than are specimens or images). In the remainder of this paper, unless otherwise noted use of the term "observation" is intended to represent sense (b).

\footnotetext{
${ }^{5}$ http://dublincore.org/

${ }_{6}^{6}$ Namespace http://purl.org/dc/terms/

${ }^{7}$ http://purl.org/dc/terms/creator
} 


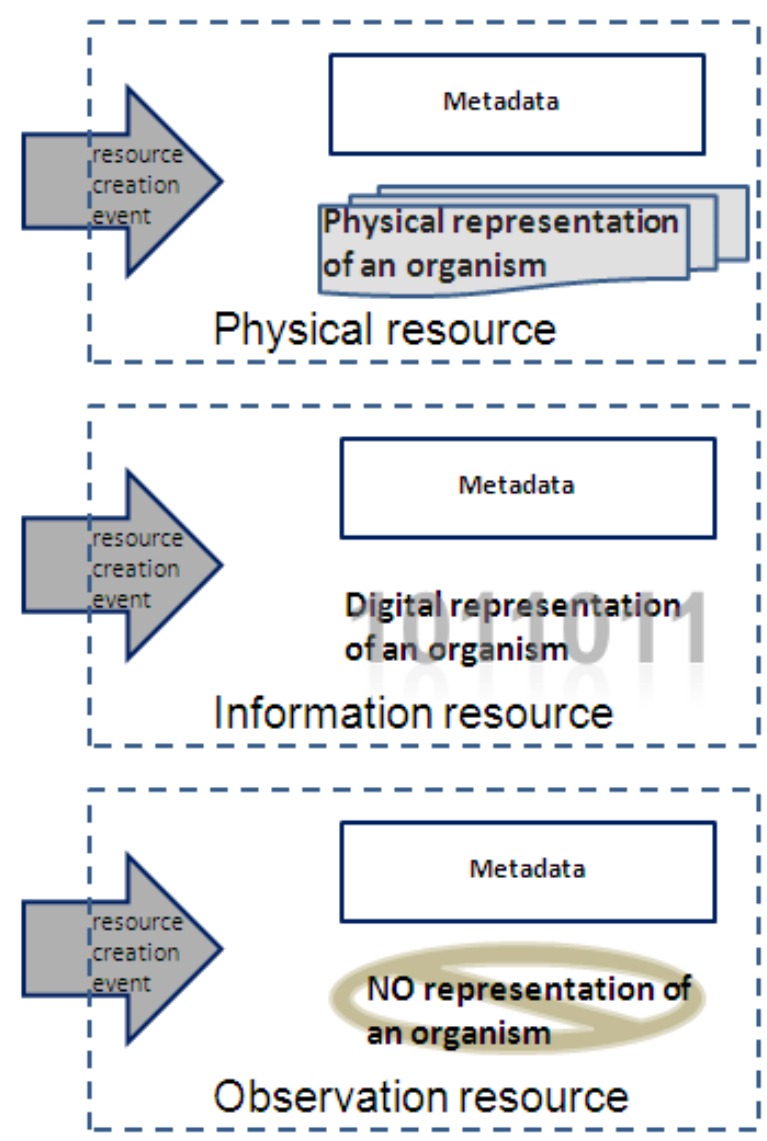

Fig. 2. Comparison of the components of the three general categories of occurrence resources.

\section{ORGANISM REPRESENTATIONS ASSOCIATED WITH OCCURRENCE RESOURCES}

Occurrence resources fall into three of the general categories listed in the definitions section based on the nature of the way in which they represent the organism (Fig. 2, Table 1). These categories are natural divisions that are based on whether and how a consumer can retrieve a digital representation of the organism from which the resource is derived.

There are several general points regarding these categories of occurrence resources. Regardless of whether a digital representation is ultimately available, all three of these types of resources have associated metadata and they have many metadata terms in common. Thus despite their differences in representation, metadata from these three types of resources can be combined in the same database.

It should also be stated explicitly that in the case of physical resources, it is desirable to create information resources that provide Internetdeliverable representations of the material artifacts. This is generally recognized and much effort has been expended toward digitization of physical resources. Thus it follows that a conceptual system describing occurrence resources must be able to relate physical resources to information resources that are derived from them.

The definition of an observation resource given here is a functional one, i.e. if an occurrence resource can never ultimately provide a digital representation of the organism, then it is an observation resource. This definition is consistent with other definitions of observations that are not explicitly based on the ultimate result of an HTTP GET request. The Biodiversity Information Standards (TDWG) Observations Task Group provides a working definition of observational data:

"... the outcomes of acts of measurement using particular protocols within the context of any objective scientific measurement activity. Examples include data from survey or monitoring efforts, controlled experiments, and sensor-derived measurements. In each case, the basic or atomic notion of an observation represents the outcome of some measurement taken of a defined attribute or characteristic of some 'entity' (e.g., an organism 'in the field', a specimen, a sample, an experimental treatment, etc.), within some context (possibly given by other observations). Every observation entails the measurement of one or more properties of some real-world entity or phenomenon." ${ }^{8}$

This definition makes two major points. One is that object of observations includes real world entities, including organisms in the field, specimens, and samples. The other is that observations measure defined attributes or characteristics. To place these statements in the formal language of URI-identified resources, observations establish values of properties that describe resources which can include occurrences (sensu Darwin Core). An important implication of this is that as defined here, observation resources record metadata (i.e. data about resources that can be described using language such as Resource Description Framework, RDF) and not data in the sense of what is returned in the resolution of a

\footnotetext{
${ }^{8}$ http://wiki.tdwg.org/Observational/
} 
Table 1. Categories of occurrence resource and their associated representations

\begin{tabular}{|l|l|l|l|}
\hline resource category & $\begin{array}{l}\text { how an organism } \\
\text { is represented by } \\
\text { that category }\end{array}$ & specific representations & $\begin{array}{l}\text { how a consumer can } \\
\text { acquire a representation of } \\
\text { the organism }\end{array}$ \\
\hline $\begin{array}{l}\text { information } \\
\text { resource }\end{array}$ & by a digital file & $\begin{array}{l}\text { digital image } \\
\text { digital audio } \\
\text { digital video } \\
\text { DNA sequence }\end{array}$ & $\begin{array}{l}\text { HTTP GET returns data } \\
\text { which is the representation } \\
\text { in digital form }\end{array}$ \\
\hline physical resource & $\begin{array}{l}\text { by a material } \\
\text { artifact }\end{array}$ & $\begin{array}{l}\text { film photograph } \\
\text { film movie } \\
\text { audiotape sound recording } \\
\text { preserved specimen } \\
\text { living specimen } \\
\text { seed } \\
\text { tissue sample }\end{array}$ & $\begin{array}{l}\text { HTTP GET does not return } \\
\text { data, but a redirect may } \\
\text { an information resource that } \\
\text { is a representation derived } \\
\text { from the artifact }\end{array}$ \\
\hline $\begin{array}{l}\text { observation } \\
\text { resource (kind of } \\
\text { defined abstract } \\
\text { resource) }\end{array}$ & no representation & none & $\begin{array}{l}\text { HTTP GET returns nothing. } \\
\text { No redirect can result in } \\
\text { return of a representation. }\end{array}$ \\
\hline
\end{tabular}

GUID. This concept is explicitly stated in Pereira (2009) which states that "core objects in biodiversity information systems such as ... observations are not associated with immutable sequence of bytes that could be returned in the LSID getData() call. It is reasonable not to return anything in the getData() call." This concept of observation resources places them in the category of abstract resources (because they are not associated with a corresponding material object), although not in the category of conceptual resources (because the observation is a defined entity that is not subject to interpretation). This concept of an observation is also narrower than the general category of "observational data" as defined by Kelling (2008).

The TDWG Observation Task Group definition of "observation" also includes metadata resulting from measurements of specimens and samples. However, in the system for categorizing resources presented here, such observations would not themselves be considered to be metadata for separate observation resources, but rather metadata associated with other resources that are physical. By the functional definitions in Table 1, a numeric measurement of an organism in the field (e.g. a measured bill length of a bird) could be considered an information resource if the measurement is considered a very simplified representation of the organism and is an immutable series of bytes (i.e. data as opposed to metadata which are subject to change). The important point is that the functional categorization of the three general types of Occurrence resources determines the kinds of metadata that one should expect for them and the way that those metadata should be organized.

\section{METADATA ASSOCIATED WITH ALL OCCURRENCE RESOURCES}

Despite the differences among the three general categories of occurrence resources, they all have several properties in common. The metadata records for all occurrence resources should include a globally unique identifier (dwc:occurrenceID), information about who created the resource (dcterms:creator), information about when the resource creation event occurred (dcterms:created), information about where the resource creation event occurred (location information), and information about the particular nature of the resource. This summarizes in the most basic way the "who, what, where and when" 
desired by the Global Biodiversity Information Facility (GBIF) ${ }^{9}$.

Recently, much effort has been exerted to establish precise location information for occurrence records, i.e. to geolocate them (Chapman and Wieczorek 2006). The ultimate goal of this effort is to create descriptors of location that are unambiguous and mappable by software. For newly created occurrence resources or those which have been precisely geolocated, the following set of four Darwin Core terms $\left(\mathrm{dwc}:{ }^{10}\right)$ unambiguously define the location where the occurrence occurred: dwc:decimalLatitude, dwc:decimalLongitude, dwc:geodeticDatum, and dwc:coordinateUncertaintyInMeters ${ }^{11}$. The first two terms define the numeric coordinates of the location, the third term describes the reference system used (for GPS measurements the default reference system is WGS84 which has the EPSG code of EPSG:4326 ${ }^{12}$; "unknown" is also a valid value), and the fourth term provides an estimate of the uncertainty of the measurement. A fifth term, dwc:locality, provides a verbal description of the location at the lowest geographic level. A locality description is important because it can make it easier for an observer on the ground to return to the location and because it can be used to validate the coordinates (Chapman and Wieczorek 2006). Darwin Core also provides many other terms to describe location using large-scale geographic and political subdivisions and these descriptors may be included in an occurrence record along with the five terms listed above. Since the values of those additional terms may be subject to change over time as political boundaries and names change, and because they can theoretically be generated by software from the four basic mathematical terms, they should be considered of secondary importance. However, for older pre-existing records it may be difficult or impossible to establish a precise location for the occurrence. In such cases, the additional terms may be the only means available for specifying location.

A description of the nature of the resource is important in order to help potential users of the metadata to assess the fitness of the resource for

\footnotetext{
${ }^{9}$ http://www.gbif.org/

${ }^{10}$ Namespace http://rs.tdwg.org/dwc/terms/

${ }^{11}$ http://code.google.com/p/darwincore/wiki/Location\#verbatimLati tude,_verbatimLongitude

12 http://spatialreference.org/
}

their intended use. There are three terms that can be used to specify the nature of a particular resource. The property rdfs:type ${ }^{13}$ has been designated as the means by which the nature of the biodiversity resource should be specified to consuming applications (example given in Pereira et al. 2009). Since resources should be typed according to the TDWG ontology "or other accepted vocabularies" (Richards 2009), the TDWG ontology class TaxonOccurrence ${ }^{14}$ is the appropriate value of rdfs:type for occurrence resources. The term dcterms:type ${ }^{15}$ is well known beyond the biodiversity community and has a defined type vocabulary ${ }^{16}$. The values PhysicalObject, StillImage, MovingImage, and Sound are appropriate for occurrence resources. However, there is no dcterms:type vocabulary term that adequately describes observation resources. The property dwc:basisOfRecord ${ }^{17}$ provides a more specific description of the resource than dcterms:type and uses a defined type vocabulary $^{18}$ that is designed particularly for the biodiversity community. Those terms describe many of the kinds of resources that are occurrences and include PreservedSpecimen, FossilSpecimen, LivingSpecimen, HumanObservation, and MachineObservation.

\section{METADATA ASSOCIATED WITH SPECIFIC CATEGORIES OF OCCURRENCE RESOURCES}

Physical resources are distinguished from other occurrence resources by the physical artifact that represents the organism. Therefore, the metadata that are most relevant to a physical artifact answer the question "How can I find/look at/get the artifact?" In a biodiversity context, physical artifacts are generally a part of museum/herbarium/botanical garden/zoo collections, so all of the terms related to where and how these artifacts are stored in a collection are relevant exclusively to physical resources.

Information resources are the only category of occurrence resources where the representation

\footnotetext{
${ }^{13}$ http://www.w3.org/2000/01/rdf-schema\#type

${ }^{14}$ http://rs.tdwg.org/ontology/voc/TaxonOccurrence\#TaxonOccurre nce

$15 \underline{\mathrm{http}}$ ///dublincore.org/documents/dcmi-terms/\#terms-type

16 DCMI Type Vocabulary section of http://dublincore.org/documents/dcmi-terms/

${ }_{17}$ http://rs.tdwg.org/dwc/terms/index.htm\#basisOfRecord

${ }^{18}$ http://rs.tdwg.org/dwc/terms/type-vocabulary/index.htm
} 
itself can be stored and distributed electronically. Thus, the foremost requirement for a digital resource is that the digital representation exists as data (i.e. a retrievable file of immutable bytes) from at least at one location in the Internet. In addition to the URL from which the data can be retrieved, metadata terms that specify the format of the data and the circumstances under which the information resource can be used (i.e. copyright, licensing, and attribution information) are important. The TDWG/GBIF Multimedia Resources Task Group (MRTG) ${ }^{19}$ draft standard metadata schema ${ }^{20}$ is appropriate for occurrence information resources and its recommendations should be followed.

Since observation resources have no representation associated with them, there are no specific metadata terms required to describe the representation as is the case for physical and information resources. Since the focus of most observations is measurement data, specifying the properties, format, and units of the measurements will be important aspects of observation resources metadata.

\section{OCCURRENCE RESOURCE CREATION NETWORKS}

General patterns in occurrence resource creation In the previous section it was established that various categories of occurrence resources are created from other resources during resource creation events and that it is sometimes desirable to create other occurrence resources from those occurrence resources. These relationships can be diagrammed generally by Fig. 3 .

The following points can be made about the relationships among occurrence resources:

1. Every occurrence resource must have a resource from which it is derived during a resource creation event.

2. Every occurrence resource is the result of a single resource creation event.

3. An occurrence resource may serve as the source of one or more derivative occurrence resources, or it may have none.

4. An occurrence resource may be associated with a physical/digital representation of an organism. If it has no representation, it is considered in this context to be an observation resource.

\footnotetext{
${ }^{19} \mathrm{http}: / /$ www.keytonature.eu/wiki/MRTG

${ }^{20} \mathrm{http://www.keytonature.eu/wiki/Submission \_ v0.9}$
}

5. An occurrence resource which is an information resource may be derived from either a physical resource or another information resource.

6. An occurrence resource which is a physical resource will only be derived from another physical resource. (There may be trivial examples where a physical resource could be derived from an information resource, such as a paper print of a digital image. But since a general goal is to create distributable electronic representations of physical representations, information resources generally reside at the end of a chain of resource creation.)

7. An occurrence resource which is an observation resource cannot be derived from a representation associated with an information or physical occurrence resource. If one were to make "observations" of a physical or information occurrence resource (e.g. to take measurements from a specimen or image) those "observations" would be metadata that should be associated with the representation, and should not be considered a separate observation occurrence resource. Thus observation resources can only be derived directly from an organism.

8. Information or physical occurrence resources cannot be derived from observation resources because a digital or physical representation cannot be created from something that has no representation.

\section{INDIVIDUALS AS THE ULTIMATE SOURCE OF OCCURRENCE RESOURCES}

Although an occurrence resource can be derived from another occurrence resource, ultimately all occurrence resources must originate through their chain of derivation back to an organism in its environment. I call this original organism an "individual" because it plays the role described in the definition of the Darwin Core term dwc:individualID ${ }^{21}$, "an identifier for an individual or named group of individual organisms represented in the occurrence. Meant to accommodate resampling of the same individual or group for monitoring purposes. May be a global unique identifier... ". In clonal species or small species such as mosses, it may be difficult to determine whether samples collected at a

\footnotetext{
${ }^{21}$ http://rs.tdwg.org/dwc/terms/individualID
} 


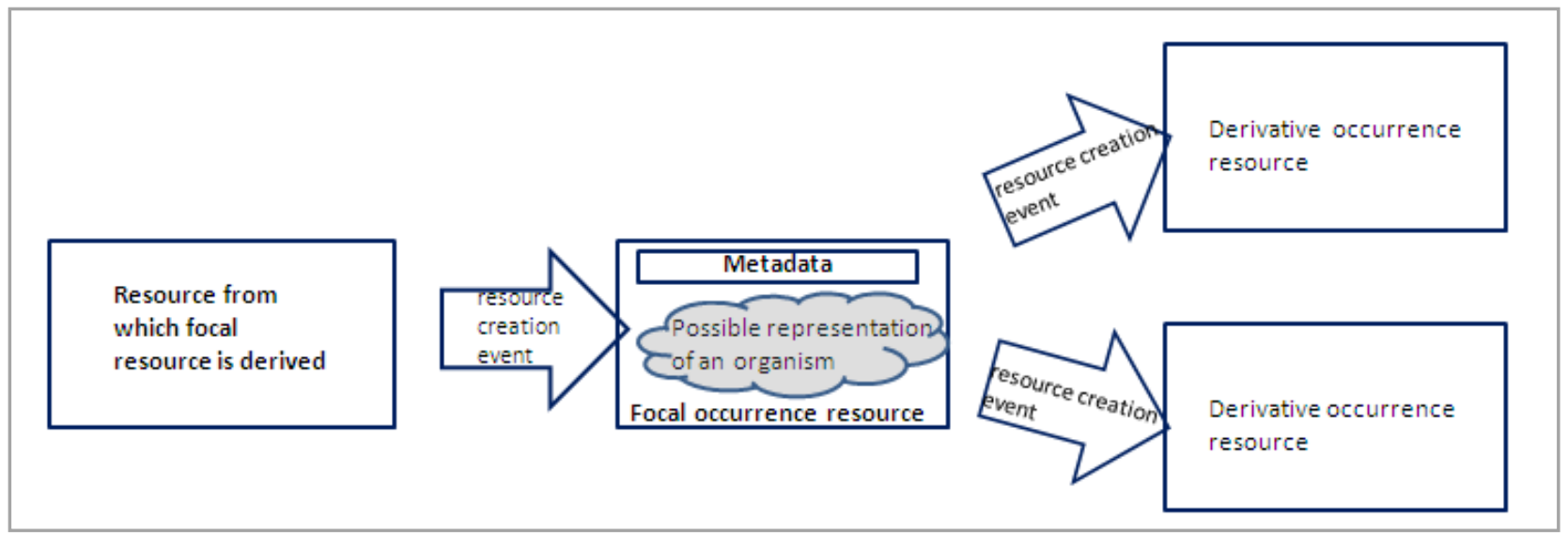

Fig. 3 Generic relationships among a focal occurrence resource and other resources related to it

particular location were collected from a single organism or a small population of organisms of the same species. Thus a group of organisms of the same species in the same location may be considered an "individual" even if technically they are several individual organisms.

If an individual were always represented by a single occurrence resource, then considering the individual to be a separate resource would be superfluous. Properties related to taxonomic identity could as easily be associated with the occurrence resource as they could be associated with the individual. However, it is possible for a single individual to be observed, sampled, or imaged multiple times or to be represented by several different types of occurrence resources. It would be redundant to assign the same taxonomic identity metadata to multiple occurrence resources derived from the same individual rather than to assign those metadata to the individual and then link the occurrence resources to the individual. In addition, it would be inconsistent to make a determination of one taxonomic identity for one occurrence resource and make a different determination of identity based on and assigned to another occurrence resource derived from the same individual. That individual can only have a single identity, so any determinations assigned based on one resource should apply to all other resources derived from the same individual. The most straightforward way to ensure this is to assign the determinations to the individual rather than to the separate occurrence resources.
The resource representing a wild individual can also connect determinations to resources derived from organisms other than that individual if those organisms arise from the individual through cloning (e.g. vegetative propagation) or even sexual reproduction. Those additional derivative organisms (which generally would generally not be in their natural environment and would therefore be classified as living specimens) must have the same taxonomic identity as the wild individual from which they originate, so any determination based on a derivative organism must apply to the individual itself and all other organisms derived from it.

\section{SOME EXAMPLES OF RESOURCE CREATION NETWORKS}

The following four figures illustrate some examples of increasingly complex networks of resource creation.

Fig. 4 illustrates the types of resources involved in a classical museum collection. In this situation an herbaceous plant is collected in its entirety, so when the preserved specimen is created, the individual ceases to exist in the environment. It is easy to consider the plant and the specimen to be one and the same thing. Because of the simplicity of this network, a simple and flat table in a database can be used to compile the data/metadata associated with specimens of this type. In a typical herbarium, the specimen is assigned a taxonomic identity (name) and the 


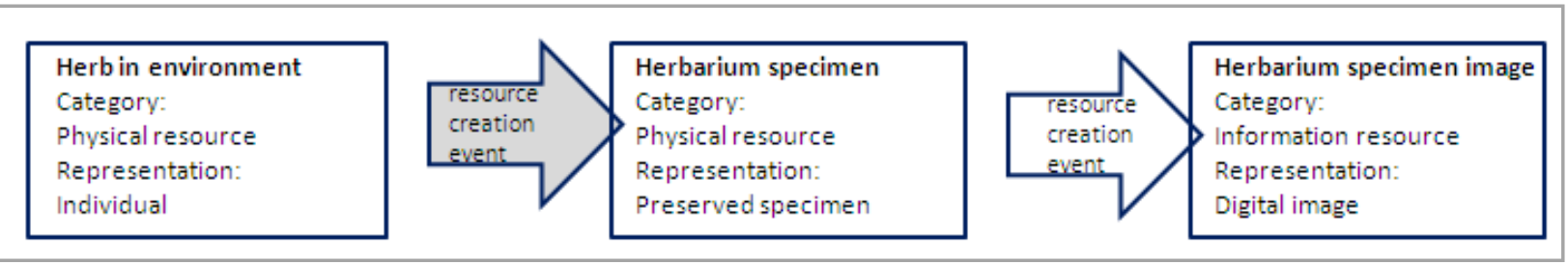

Fig. 4. Diagram showing the relationships among an individual, resources derived from it (a specimen and a specimen image), and the resources' associated creation events in a classic museum collection scenario.

plant's location is noted in the database for the specimen. Later, the specimen is imaged and a single reference to the image is made in the database record for the specimen. In such a scenario, there is little benefit to considering the individual to be a separate resource because nearly all relevant metadata can be associated with the specimen.

In Fig. 5, an insect is located in a field survey. Before the insect is collected and killed, it is photographed live in its environment with a digital camera. After the survey trip, the insect is pinned and added to the museum's collection. As a part of the museum's efforts to document its collection, the pinned specimen is imaged digitally. The museum is also supporting a molecular systematics effort and donates a small sample from the insect from which DNA is extracted, sequenced, and submitted to GenBank. Later, as a part of an effort to develop a database of characters, the pinned specimen is dissected andseparate specimens are created of various body parts, which are then digitally photographed under a microscope. The original pinned specimen ceases to exist as an entity upon the creation of the separate part specimens. As they have now become a permanent part of the collection, the separate part specimens are assigned their own catalog numbers.

Fig. 6 represents a hypothetical collection scenario for a botanical garden. During a field expedition, a survey crew locates an unusual tree and takes a number of digital images of the tree. A botanist on the team reviews the images and tentatively identifies the tree as a rare species. The survey crew returns to collect cuttings for propagation and branch/leaf specimens from the tree for the botanical garden's herbarium. Another team returns later in the season after fruit has set and collects seeds. Part of the seeds are imaged and stored as a part of an effort to preserve germplasm of rare species. Other seeds are germinated and the resulting plants are added to the garden's permanent collection. DNA is collected from one of the living specimens in the garden and the DNA sequence is submitted to GenBank. Additional herbarium specimens may be collected from the living specimens in addition to those collected from the original tree and other individuals may be vegetatively propagated and sent to other botanical gardens. Although the various botanical garden specimens are separate organisms from individual in the wild, those living specimens are derived clonally or genetically from the individual in the environment and thus can provide information regarding the wild individual's taxonomic identity.

Fig.7 involves an effort to track an individual of a large, rare species of bird (e.g. whooping crane). The young bird is initially observed visually in the nesting ground and recorded in an ornithologist's field records. Several days later the decision is made to capture and band it. The following year, the information from the band is recorded by a different ornithologist in the overwintering grounds. At a later time the bird is recaptured. A tissue sample is collected for DNA extraction and analysis and the bird is fitted with a radio tracking device. Data/metadata are collected throughout migration using telemetry from the tracking device.

Historically, biodiversity records have been organized around specimens. In a simple scenario such as Fig. 4, this is practical. However, in Figs. $5-7$, occurrence information resources (i.e. images) are collected directly from the individual and are not derived from any specimen. In Fig. 7, no specimen is collected at all. Thus in any collection scenario more complicated than Fig 5 it makes sense to consider the individual as an identified resource (i.e. having an assigned GUID). 


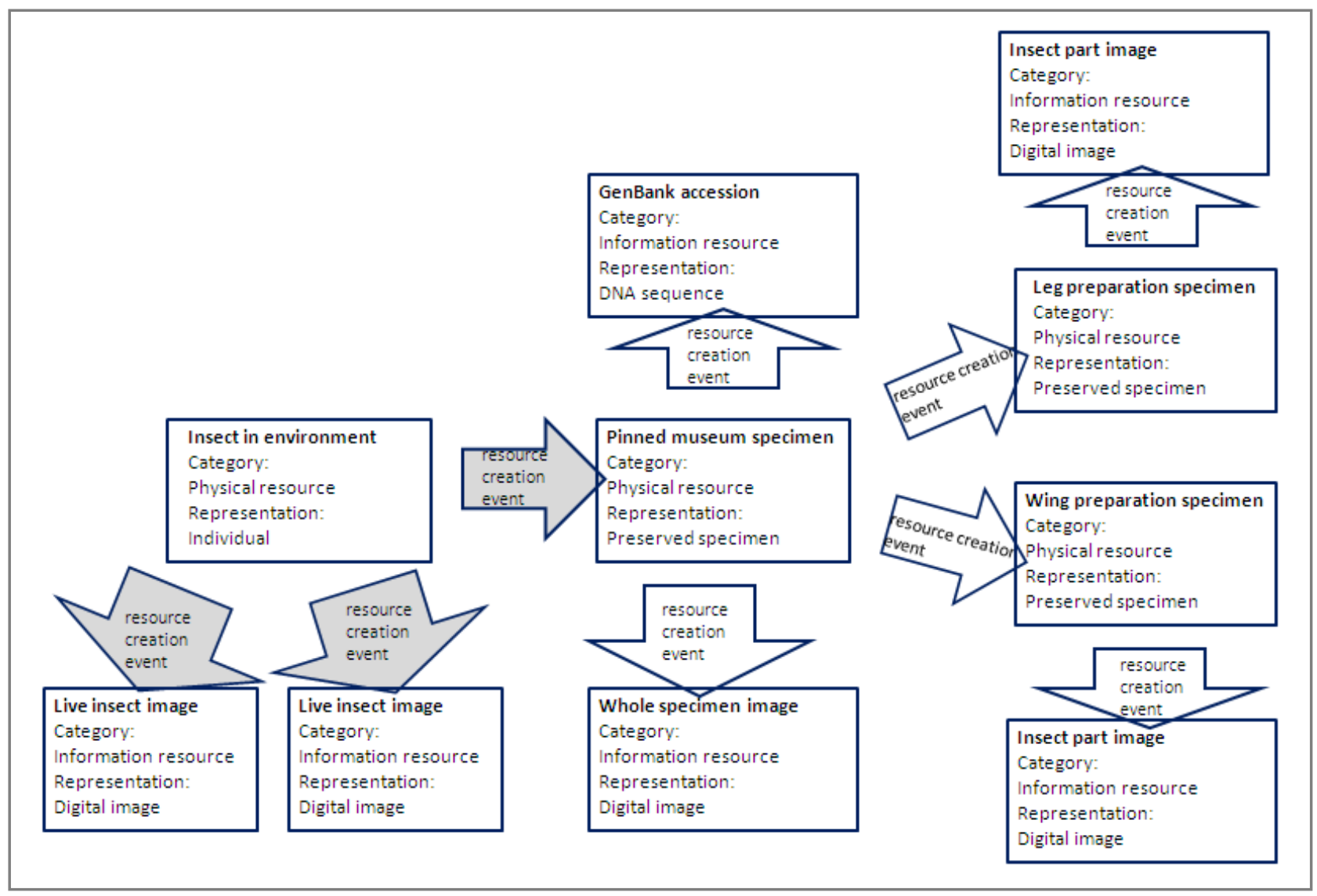

Fig. 5. A more thorough museum collection scenario involving an insect specimen.

\section{METADATA ASSOCIATED WITH INDIVIDUALS}

In previous sections it was noted that metadata associated with occurrence resources describes where, when, and by whom the resource was created as well as the nature of the resource itself and how any representations associated with the resource can be accessed. Since an individual is by definition in its natural state (any individual taken out of its natural state would become a living specimen), no human entity can be assigned as its creator.

Although an individual could be considered to have had a time of creation (i.e. when it was born, hatched, or germinated), it is not possible to know when nor where this occurred without creating an occurrence resource. If the time of the organism's birth were noted by a human observer, then an observation resource would be created with the time of birth represented by the resource creation event metadata for the observation resource. If the birth were digitally imaged, then an information resource (StillImage) would be created. It is, in fact, not possible to know anything about any time of existence or location of an individual without an occurrence resource creation event occurring. For this reason, particular dates and locations of events should not be assigned to an individual. However, it would be possible, if desired, to assign to the individual the set of dates and locations for all resource creation events resulting in resources derived directly from the individual. Such a set can be considered to define the spatial and temporal range of that individual. In Figs. 4-7, such resource creation events originating directly from individuals are highlighted in gray.

In addition to the conceptual reason why location should be associated with derived occurrence resources rather than the individual organism, there are also several practical reasons. Foremost among these is that some organisms move, and since some resource creation events (those creating observations and images) do not 


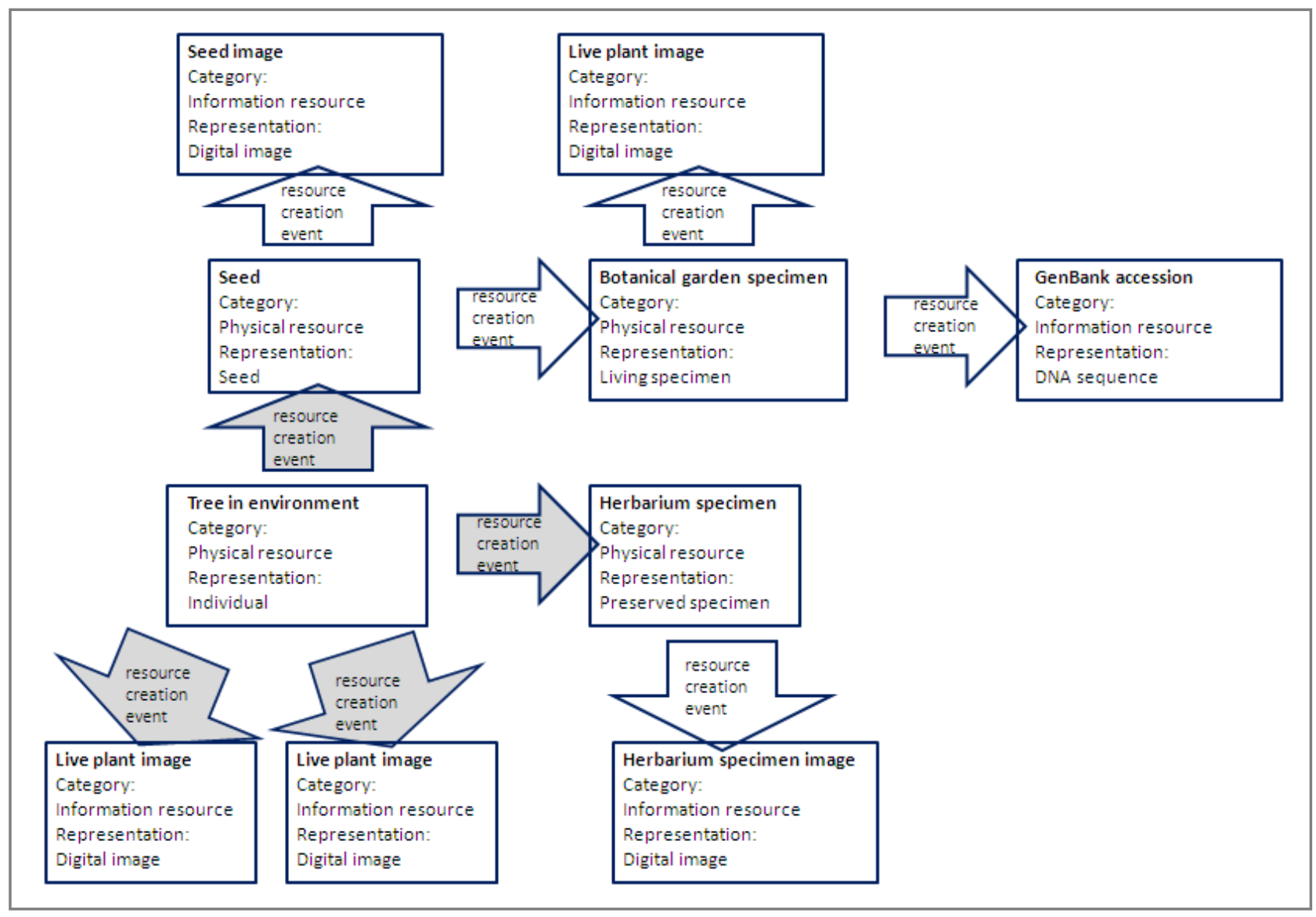

Fig. 6. Collection scheme for a botanical garden.

necessarily result in the destruction of the organism and the fixing of its location at the point of death, it may not be possible to assign a single location to a mobile organism. Therefore, the set containing all locations where occurrence resources were created directly from an individual represents the geographic range of that moving individual.

It also makes sense to record multiple resource creation locations even for sessile organisms. For example, an individual plant may be imaged several times by a GPS-enabled digital camera. Because of inaccuracies in the determination of latitude and longitude recorded with the images, each location recorded by the camera represents an estimate of the actual location of the individual. In that situation, the set containing all locations where resource creation events occurred allows determination of a sort of "standard error" of location. It is also possible that locations associated with the creation of different resources from the same individual may have different precisions. For example, a specimen may first be collected from a tree and the tree's location determined by reference to a topographic map. At a later time, a digital image may be taken of the same tree with the tree's location determined by GPS. The precision of the GPS determination is likely to be greater than the determination from the map, so it would be important to maintain both sets of geographic coordinates along with their precisions so that a user could assess the relative utility of each for re-locating the tree.

For the reasons given above, it is clear that location is logically an aspect of the creation event of each particular occurrence resource. However, it should be noted that since some occurrence resources may not be directly derived from an individual organism in its natural state, it cannot necessarily be inferred that an occurrence resource creation event contributes to documenting the distribution of a taxon. For example, a preserved specimen may not indicate the presence of a taxon representative at the place of its creation if it was collected from a living specimen in a botanical garden. A digital still image may not indicate the 


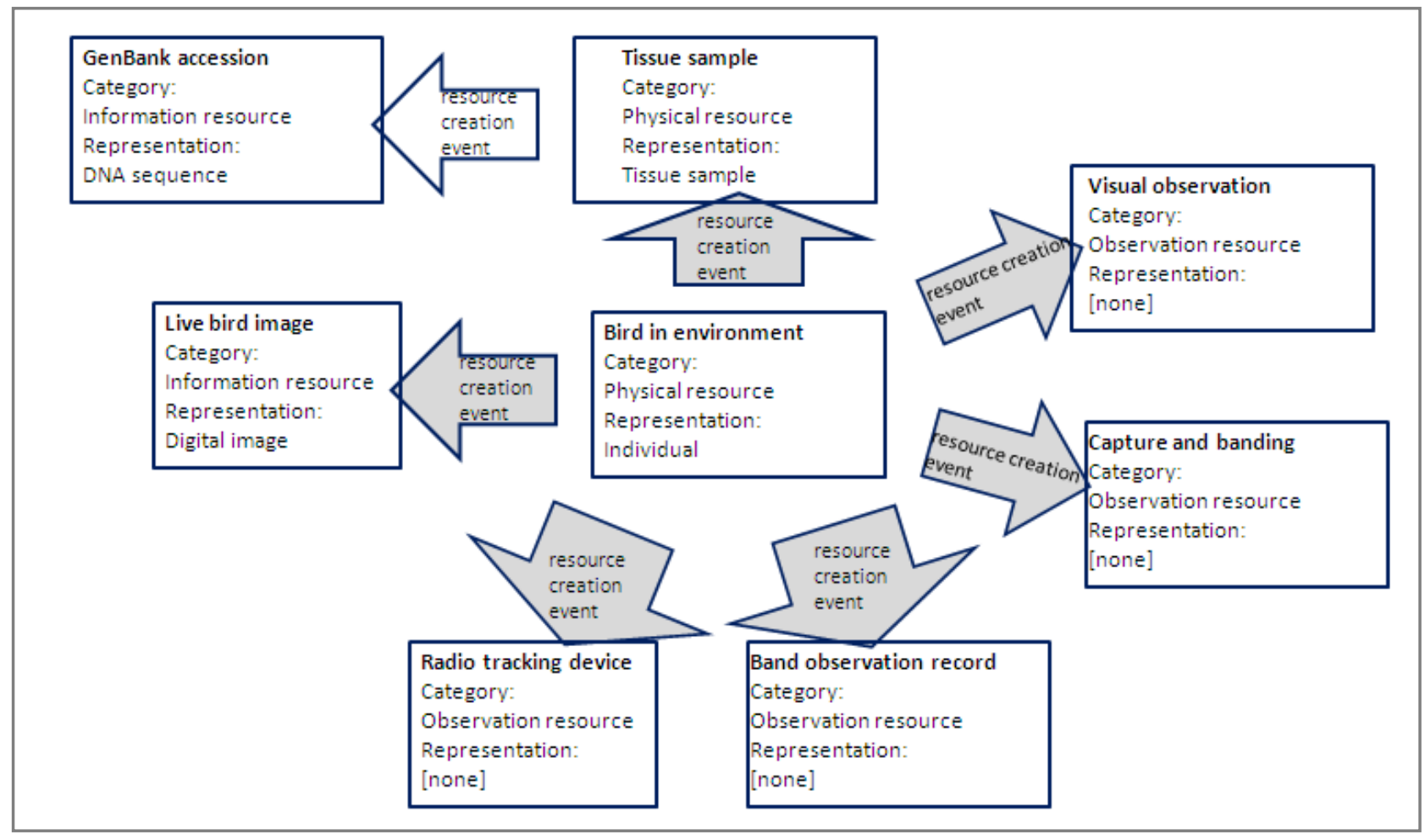

Fig. 7. Bird survey based primarily on observations.

presence of a taxon representative if it is an image of a specimen, while it may if it is a picture of a living individual in the wild. There is currently no term in the Darwin Core standard that is suitable for indicating unambiguously that the date and location of an event serves to document a taxon distribution. It can be established from the metadata that an event asserts that a representative of a taxon was present at the location of an occurrence resource creation event if the identifier for resource from which occurrence is derived is the same as the identifier of the individual that the occurrence represents. However, to serve the purpose of clarifying whether the resource creation event of an occurrence documents the distribution, the term sernec:documentsDistribution ${ }^{22}$ has been defined.

It should also be made clear that the typing of an occurrence resource using dcterms:type, rdfs:type, or dwc:basisOfRecord does nothing to restrict fitness of use of that resource to any particular purpose. Regardless of whether or not an occurrence resource is used to establish that a

\footnotetext{
${ }^{22}$ http://bioimages.vanderbilt.edu/rdf/terms\#documentsDistribution ; appropriate text values are "true" or "false"
}

taxon representative is present at a location, there is no restriction prohibiting that occurrence resource from simultaneously being used for any of a number of purposes, e.g. to define a character, as a learning tool, to illustrate a habitat, to serve as a type specimen, etc. Fitness of use for such purposes may be noted through the inclusion of appropriate properties in the metadata for the occurrence resource - there is no limit to the number of such properties that may be included.

It is useful at this point to differentiate dcterms:creator from the Darwin Core term dwc:recordedBy. As discussed in the section on resource creation events, in the context of occurrences, dcterms:creator represents the entity that caused any occurrence resource to come into existence. dwc:recordedBy is a "list ... of names of people, groups, or organizations responsible for recording the original occurrence". Thus dwc:recordedBy carries the additional connotation that the resource created by creator of an "original occurrence" (i.e. a resource created directly from an individual -- a resource creation event represented by a gray arrow in Figs. 4-7) can be used to establish the presence of a taxon at a 


\section{BASKAUF - ORGANIZATION OF OCCURRENCE-RELATED BIODIVERSITY RESOURCES}

location. Thus occurrence resources derived directly from individuals (sernec:documentsDistribution value="true") will usually have values for both dcterms:creator and dwc:recordedBy, while resources not derived directly from individuals (sernec:documentsDistribution value="false") will have no value for dwc:recordedBy. The same criteria hold for dcterms:created and dwc:eventDate. If a resource is derived directly from an individual, it should be given identical values for dcterms:created and dwc:eventDate, while a resource not derived directly from an individual should only be assigned a value for dcterms:created.

The only general category of metadata that should logically be associated with individuals is information related to taxonomic determinations. As we shall see later, there is benefit to considering these determinations as separate resources rather than as metadata of the resource representing the individual. Thus individuals as resources serve primarily as nodes that connect their derivative occurrence resources to taxonomic determinations. With few exceptions, the metadata for resources representing individuals will consist primarily of statements indicating the relationship of that individual to other resources. One such exception would be assignment of a value for dwc:establishmentMeans to indicate whether the individual was native, naturalized, adventive, or cultivated.

\section{RELATIONSHIPS AMONG DATA/METADATA RESOURCES Relationships Networks}

The conceptual scheme of resource creation was focused on networks which traced the series of events by which occurrence resources of various types were created from individual organisms. In this section, I will focus on the relationships among resources of various kinds and the ways those relationships can be specified.

In Fig. 8, each shape represents a resource as defined in the definition section. Each line represents an important relationship between two resources in the network. The heavy lines below the individual represent the connections established between pairs of resources by the occurrence resource creation events discussed previously. A given focal occurrence resource is connected by the line above to the resource from which it was derived. Lines below the resource connect it to occurrence resources that were derived from it.

Since taxonomic identity is a property of the individual organism, the individual should have one or more determinations which link that individual with a general taxonomic concept (Franz 2008), identified as "taxon" in Fig. 8. Each determination may be considered a unique, defined abstract resource linked to that individual and having its own assigned GUID (dwc:identificationID). The taxonomic concept (dwc: taxonConceptID) to which the determination is linked is a conceptual resource that may be applied to many determinations and which has its own HTTP URI (e.g. DeVries, 2009). The taxonomic concept itself may be linked to one or more scientific and vernacular names (not represented in Fig. 8) which may be considered separate abstract resources having complex relationships among themselves (Page 2006).

In common usage, an initial determination may be called the "identification" and subsequent determinations may be called "annotations". However, regardless of order, each determination represents a functionally equivalent assessment of the individual's identity, having properties of dcterms:created, dwc:identifiedBy, dwc:identificationRemarks, etc. Each determination has a relationship with one or more resources that served as the evidence by which the determination was made. This "determination based on" relationship is represented by the smalldashed lines. It should be noted that a determination has a particular resource (or resources) on which it is based, but particular resources do not have a particular determination that represents the identity of their source individual. The resource is a representation of the individual and the individual will have all of the determinations as possible assessments of its identity.

Since taxonomic identity is assigned to a resource through its relationship with the individual, this identity must be accessed via the individual at the root of the resource creation chain. The individual can be located by following the lines through resources up the derivation chain. However, it may be convenient to specify directly 


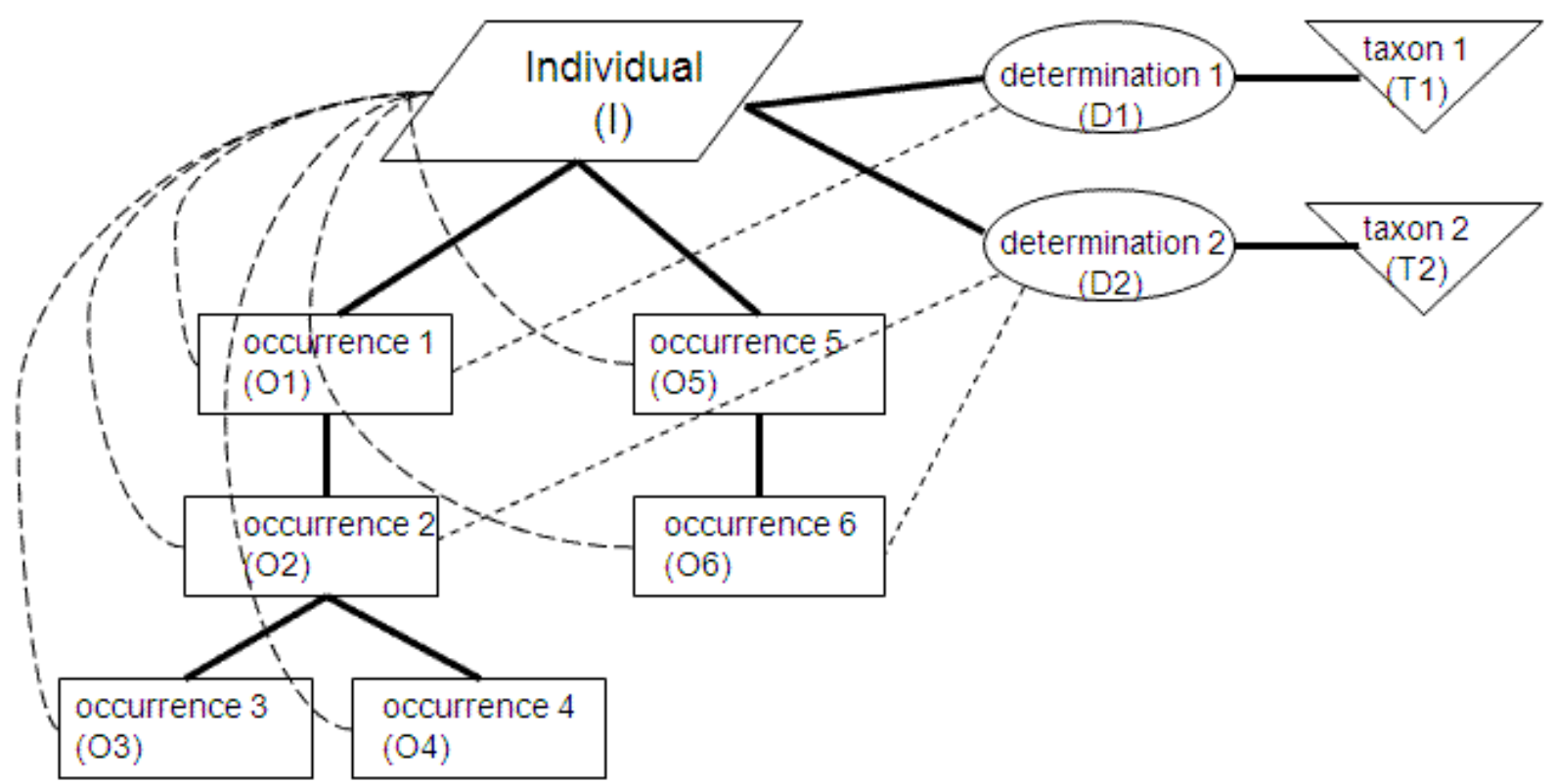

Fig. 8. Network of important relationships among resources in a complex resource creation scheme. Occurrence resources could represent some of those in Fig. 6 ( $\mathrm{I}=$ tree in environment, O1=seed, O2=botanical garden specimen, $\mathrm{O} 3=$ GenBank accession, $\mathrm{O} 4=$ =live plant image, $\mathrm{O} 5=$ herbarium specimen, O6=herbarium specimen image).

the source individual from which the resource was ultimately derived. The "source individual" relationship for each derived resource is indicated by the long-dash lines.

\section{SPECIFYING RELATIONSHIPS BETWEEN RESOURCES - RESOURCE DESCRIPTION FRAMEWORK (RDF)}

The links among resources discussed in the previous section can be defined formally using Resource Description Framework (RDF) ${ }^{23}$, which has a well-defined syntax for representing resources and relationships. RDF can be represented several ways ${ }^{24}$. Relationships can be shown as graphs, while RDF serialized as $\mathrm{XML}^{25}$ is also the default means for describing metadata when a GUID is resolved (Richards 2009).

Figs. 9-11 show RDF graphs for examples of three of the major types of resources diagrammed in Fig. 8 (the fourth, taxonomic concept, is beyond the scope of this paper). In these graphs, ovals represent resources named by HTTP URIs.

\footnotetext{
${ }^{23}$ http://www.w3.org/RDF/

24 http://www.w3.org/TR/rdf-primer/

${ }^{25}$ http://www.w3.org/XML/
}

Rectangles represent literals (composed of Unicode characters). The resource in the center of the graph is the subject of the relationships. Each arrow in the graphs represents the nature of the relationship (referred to as a "predicate" in RDF) with a description of that relationship next to the arrow. The objects of the relationships (shapes pointed to by the arrows) are the metadata associated with the subject (the object of the relationship).

In many cases, the predicate describing the relationship is a property that is a term from a well-known vocabulary. In particular, the following prefixes are used for qualified namespaces representing these vocabularies.

$$
\begin{aligned}
& \text { dcterms="http://purl.org/dc/terms/" } \\
& \text { dwc="http://rs.tdwg.org/dwc/terms/" }
\end{aligned}
$$

In addition, there are several terms for relationships described in this paper but which have not previously been defined. These terms have been formally defined in RDFS $^{26}$ and they have been assigned to the qualified namespace:

\footnotetext{
${ }^{26} \mathrm{http}: / /$ bioimages.vanderbilt.edu/rdf/terms
} 


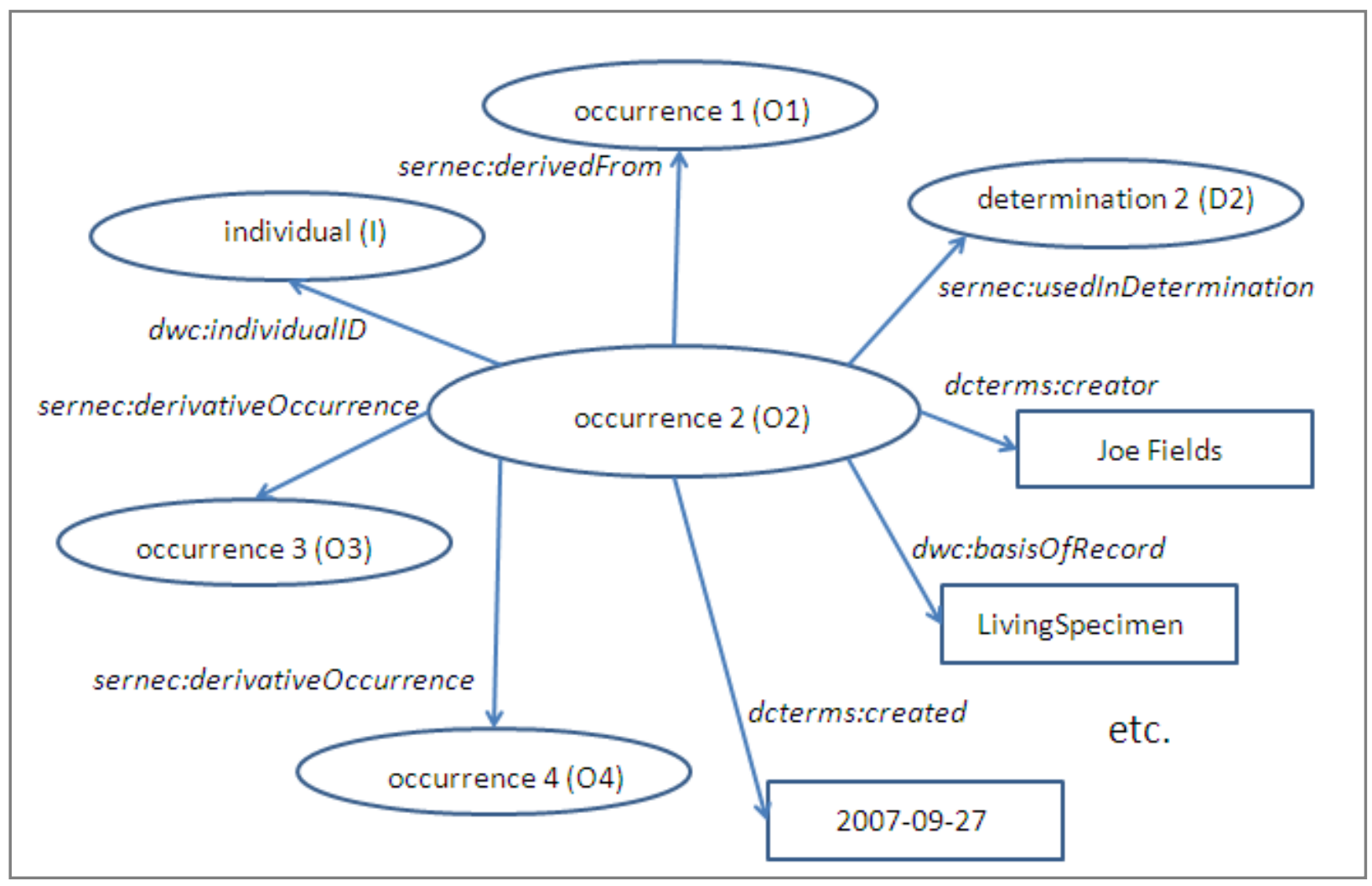

Fig. 9. RDF graph for an occurrence resource (based on relationships outlined in Fig. 8)

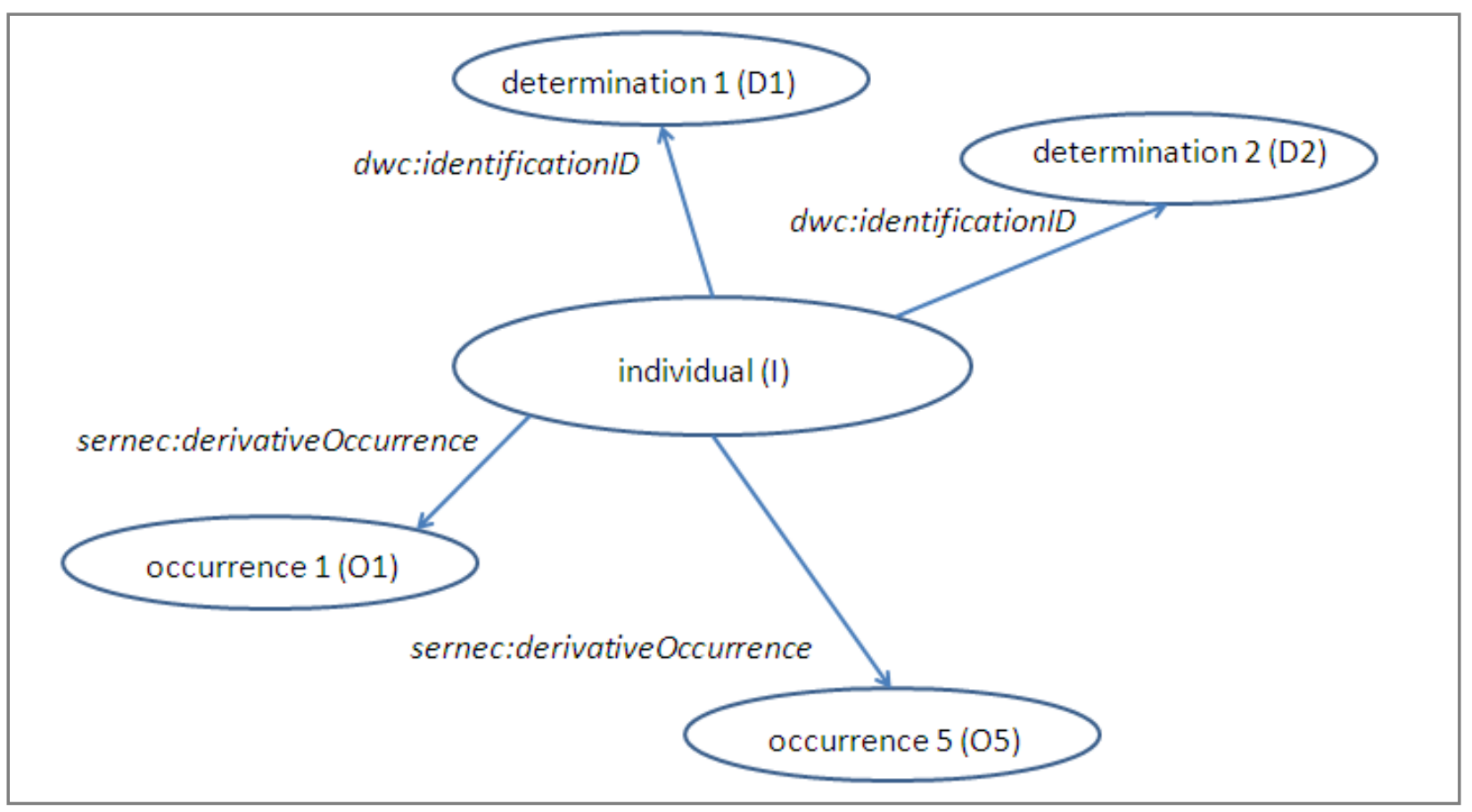

Fig. 10. RDF graph for an individual (based on relationships outlined in Fig. 8) 


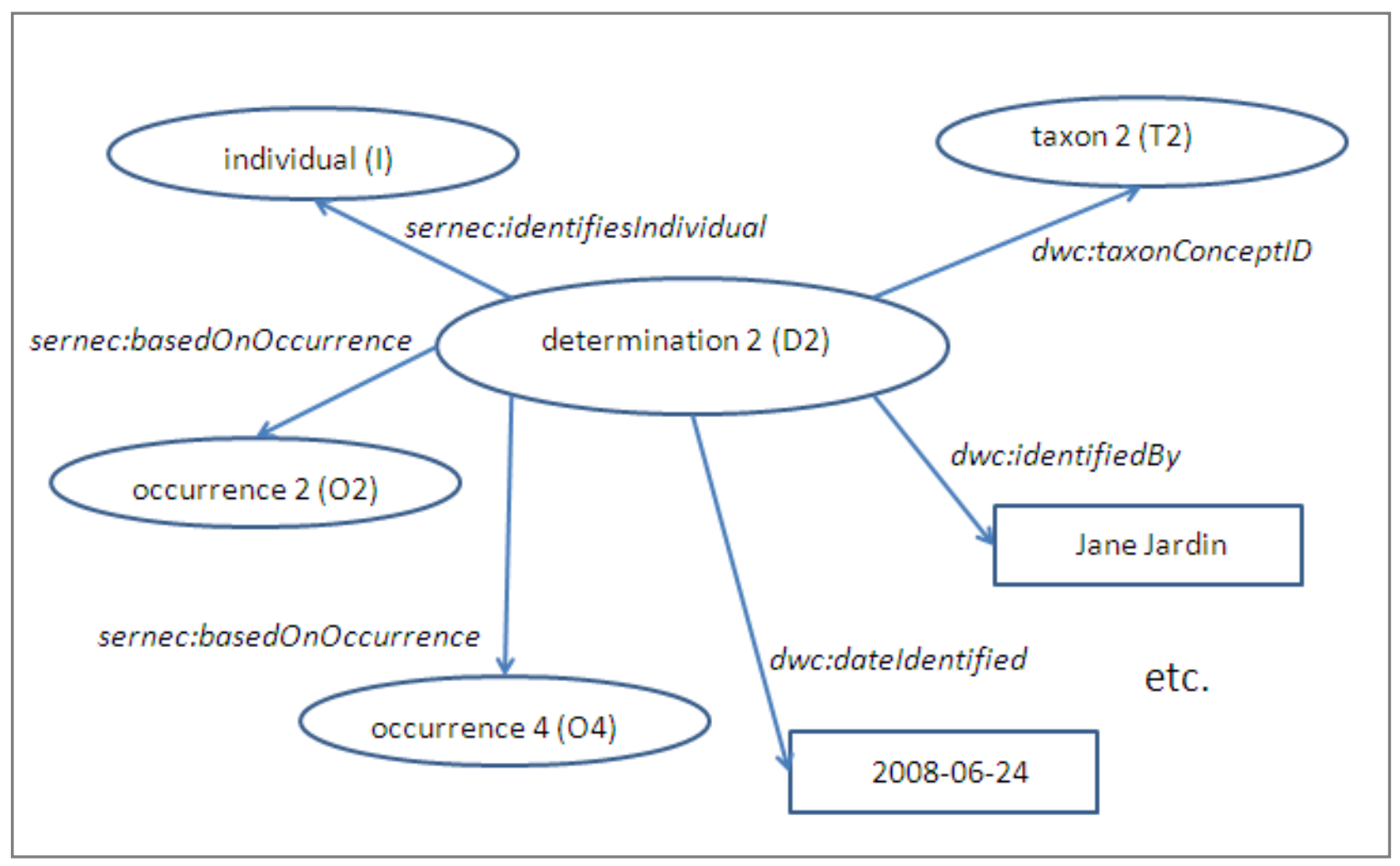

Fig. 11. RDF graph for a determination (based on relationships outlined in Fig. 8) ms\#"

sernec="http://bioimages.vanderbilt.edu/rdf/ter

These terms are:

derivedFrom Indicates the resource from which the subject (an occurrence resource) was derived. May be an individual or another occurrence resource

derivativeOccurrence Indicates an occurrence resource that was derived from the subject (the subject may be an individual or an occurrence resource)

identifiesIndividual Indicates an individual that is identified by a determination

basedOnOccurrence Indicates an occurrence resource used as the basis for a determination.

usedInDetermination Indicates a determination for which the subject (an occurrence resource) was used as a basis for identification

The properties of an occurrence (Fig. 9; Appendix A) fall into four general categories. The first includes general descriptive metadata that applies to all resources, such as the GUID of the resource, and a dcterms:description of what it is. The second is general metadata that applies to all occurrence resources, as described in the section "Metadata associated with all occurrence resources". Other metadata terms related to occurrences may be included if necessary or desired. The third category of metadata is that which applies to the specific kind of occurrence (Table 1). In general, information resources require metadata describing intellectual property rights, and use and attribution guidelines described in the MRTG schema. Physical resources may be described using specific Darwin Core terms which apply to that type of occurrence. The fourth general category is properties that describe how the occurrence resource is related to other resources. These properties relate the resource to other resources from which it is derived or which are derived from it, determinations that are based on that occurrence, and the individual from which the resource is ultimately derived.

In the example of Fig. 9 (and its corresponding Appendix A), literals were used for the values of properties referring to entities (persons and 
institutions). However, HTTP URI references could also be used and would be preferable if they represented a "semantic web-aware" resource (such as a $\mathrm{FOAF}^{27}$ file) that can be de-referenced.

The metadata of an individual (Fig. 10; Appendix B) consists almost entirely of properties that define the relationships between the individual and resources derived from it, and the individual and determinations that assign the individual to a taxon concept. This highlights the role of an individual as a node that connects other resources. One property of the individual that is assigned a literal value is dwc:establishmentMeans, which can be used to indicate whether the individual is wild or cultivated.

The metadata of a determination includes a description of who made the identification and when. But the most important aspect of the determination metadata is a description of the connections formed by the determination between an individual and a taxon concept that is consistent with the facts of the individual as illustrated by its occurrences. This connection is supported by including links to the occurrence resources on which the determination was based. If those occurrences are information resources, a user could examine them to verify the determination.

One advantage of this system of organization is that it requires very few predicates to define the relationships among occurrence resources, individuals, and their determinations. By using generic descriptions of relationships followed by tagging $^{28}$, the overall system is simpler than the alternative of creating different relationship types for different kinds of object resources. For example, sernec:derivativeOccurrence is used rather than creating separate predicates for each type of derivative occurrence object (e.g. rather than using dwc:associatedMedia and dwc:associatedSequences specifically to indicate images and sequences derived from specimens and tissue samples). Similarly, by considering both identifications and annotations as one type of resource (determination), a single predicate can be used to indicate the relationship of another resource with the determination. If desired, an application can infer which determination is the

\footnotetext{
${ }^{27} \mathrm{http} / / / \mathrm{www}$. foaf-project.org/

${ }^{28}$ http://wiki.tdwg.org/twiki/bin/view/TAG/SubclassOrNot
}

initial identification by finding the determination with the earliest dwc:dateIdentified value.

It should also be noted that relationships involving an occurrence resource do not have to be limited to those illustrated here. For example, nodes representing habitat sites or character definitions could group occurrence resources derived from different individuals. Appropriately defined predicates could link the occurrence resources to those nodes through entries in the RDF XML file for the occurrence. The relationships outlined here represent the minimum set of relationships that are needed to establish the taxonomic identity that should be associated with the occurrence and to link the occurrence to others that are closely related to it.

\section{CONCLUSIONS \\ The role of individuals.}

Resources representing individuals serve as nodes which connect multiple occurrence resources that are derived from that same individual, such as duplicate specimens, direct images of the live organism, and multiple observations. They also provide a single resource to which determinations can be assigned, avoiding the necessity of repeating identification metadata in all of the occurrence records for the same organism and insuring that annotations are applied to all of those occurrences. One deficiency of the conceptual scheme described in this paper is that it does not adequately handle circumstances where a single occurrence resource contains evidence of multiple individuals. Examples would include the contents of a pitfall trap which included several insects, an image of a habitat showing several individuals of different species, and a specimen of an organism that showed evidence of parasitism by an individual of a different species.

\section{Clarification of artifact types and their relationships to resource classes.}

By explicitly categorizing occurrence resources according to the nature of their representation of an organism (physical, digital, or none), it becomes clearer which metadata elements are most appropriately assigned to various particular kinds of occurrence resources. This categorization also makes it clear in the process of GUID resolution what metadata should be 


\section{BASKAUF - ORGANIZATION OF OCCURRENCE-RELATED BIODIVERSITY RESOURCES}

expected by a consuming application for those kinds of resources.

\section{Clarification of what determines that an} occurrence provides information about the presence of a representative of a taxon at a location.

It is clear from Figs. 4-7 that occurrence resources assert that a taxon representative is present at a location if the occurrence resources were derived directly from the individual (i.e. gray arrows) and not because those resources are of any particular kind. This clarification can be made explicit through use of the term sernec:documentsDistribution.

\section{Role of resource creation networks in defining possible relationships among resources.}

It is a basic premise of this paper that the assignment of metadata to resources, the structuring of that metadata, and the relationships among biodiversity resources expressed in their metadata should reflect process used to collect and create occurrence resources (i.e. the resource creation network). Since this process is general and applies generically to many kinds of occurrence resources, a few general metadata structures and relationship types (illustrated in the RDF graphs and examples in Appendices A-B) can be adopted rather than creating multiple special resource classes, structures, and relationships.

\section{Applicability of this framework.}

Significant challenges remain before the promise of a worldwide network of biodiversity information can come to fruition. In particular, implementation of the GUIDs necessary for the functioning of this network is a difficult task. It is hoped that the framework outlined in this paper will facilitate the development and application of GUIDs to occurrences by clarifying what types of resources should be identified by GUIDs and which metadata terms can be appropriately used to specify the properties of those resources.

\section{ACKNOWLEDGEMENTS}

I began to think about the issues raised in this paper during an email discussion of the SERNEC (Southeast Regional Network of Expertise and Collections) Live Plant Imaging subgroup during
2007-08 (http://www.sernec.org/files/summary-ofdiscussion.pdf). In particular, the comments of Debbie Paul, Boyce Tankersley, Alexey Zinovjev, and John Wieczorek were helpful in developing my thinking as were earlier comments made by Austin Mast. Irina Kadis provided examples in the context of a botanical garden. Alexey Zinovjev, Irina Kadis, John Wieczorek, and Donald Hobern made helpful comments on drafts of the manuscript. Two anonymous reviewers provided useful comments and references.

\section{LITERATURE CITED}

Berners-Lee, T., R. Fielding, and L. Masinter, 2005. Uniform Resource Identifier (URI): Generic Syntax (Request for Comments: 3986). The Internet Society. (accessed 2009-12-15) ${ }^{29}$

Chapman, A.D. and J. Wieczorek (eds), 2006. Guide to Best Practices for Georeferencing. Copenhagen: Global Biodiversity Information Facility. (accessed 2010-01-15) $)^{30}$

Cryer, P., R. Hyam, C. Miller, N. Nicolson, É.ó Tuama, R. Page, J. Rees, G. Riccardi, K. Richards, R. White, 2009. Adoption of Persistent Identifiers for Biodiversity Informatics: Recommendations of the GBIF LSID GUID Task Group, 6 November 2009 (accessed 2009-12-15) ${ }^{31}$.

Darwin Core Task Group, 2009. Darwin Core (accessed 2009-12-15) . $^{32}$.

DeVries, P.J. 2009. GeoSpecies Knowledge Base available from http://lod.geospecies.org. (accessed 2009-12-15) $)^{33}$.

Franz, N., R.K. Peet, and A.S. Weakley, 2008. On the Use of Taxonomic Concepts in Support of Biodiversity Research and Taxonomy in Q.D. Wheeler (ed.) The New Taxonomy. The Systematics Association special volume series 76, Boca Raton: CRC Press. ${ }^{34}$

Jacobs, I. and N. Walsh (Eds.), 2004. Architecture of the World Wide Web, Volume One (W3C Recommendation 15 December 2004, accessed 2009-12-15). ${ }^{35}$

Jones, M.B., N. Bertrand, J. Holetschek, V. Hutchison, B.C. Ko, Á.Suárez-Mayorga, M. Meaux, W. Ulate, D. Watts, T. Robertson, and É.Ó Tuama, 2009. Report of the GBIF Metadata Implementation Framework Task Group (MIFTG). 16 December

\footnotetext{
${ }^{29}$ http://tools.ietf.org/html/rfc3986

${ }^{30}$ http://www2.gbif.org/BioGeomancerGuide.pdf

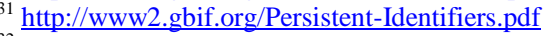

32 http://rs.tdwg.org/dwc/

${ }^{33}$ http://about.geospecies.org/

${ }_{34} \mathrm{http}$ ///www.sernec.org/files/UseTaxConcepts_0.pdf

${ }^{35}$ http://www.w3.org/TR/webarch/
} 
2009. Copenhagen: Global Biodiversity Information Facility. (accessed 2010-05-12). ${ }^{36}$

Kelling, S., 2008. Significance of organism observations: Data discovery and access in biodiversity research. Report for the Global Biodiversity Information Facility, Copenhagen. ${ }^{37}$

Neufeldt, V. and D. B. Guralnik (eds.), 1994. Webster's New World Dictionary of American English, 3rd edition. New York: Prentice Hall.

Page, R.D.M., 2006. Taxonomic Names, Metadata, and the Semantic Web. Biodiversity Informatics 3:115.
Richards, K., 2009. TDWG GUID Applicability Statement Draft Standard. Biodiversity Information Standards (TDWG) 3 September 2009 (accessed 2009-12-15). ${ }^{38}$

Pereira, R., K. Richards, D. Hobern, R. Hyam, L. Belbin, and S. Blum, 2009. TDWG Life Sciences Identifiers (LSID) Applicability Statement (draft standard). Biodiversity Information Standards (TDWG) 3 September 2009 (accessed 2009-1215). ${ }^{39}$

W3C Technical Architecture Group (TAG) 2005. httpRange-14: What is the range of the HTTP dereference function? (accessed 2009-12-15) ${ }^{40}$

\footnotetext{
${ }^{36} \mathrm{http}: / /$ www2.gbif.org/GBIF-MIFTG-Report.pdf

${ }^{37}$ http://www2.gbif.org/Observational_Data.pdf
} 
Appendix A. Sample RDF file for the occurrence resource in Fig. 9 using the example of the living specimen (O2) in the botanical garden scheme (Figs. 6 and 8). Other examples featuring an image of that specimen $(\mathrm{O} 4)$ and one of the live plant images in Fig. 6 are also provided.

The following GUIDs were used to represent resources in these examples as well as the other appendices:

http://bigbotanicalgarden.org/individual/10224=I=tree in environment

http://saveourspecies.org/germplasm/54899= O1=seed

http://bigbotanicalgarden.org/collection/25993=O2=botanical garden specimen

http://www.ncbi.nlm.nih.gov/nuccore/BQ12345= O3=GenBank nucleotide sequence, accession number BQ12345

http://bigbotanicalgarden.org/image/52011= O4=live plant image of the living specimen.

http://stateunivherbarium.org/vascular/67988=05=herbarium specimen

http://stateunivherbarium.org/vascular/67988\#img=O6=image of herbarium specimen

http://bigbotanicalgarden.org/image/44321=live plant image of the tree in environment.

http://bigbotanicalgarden.org/ individual/10224\#ABCD $=\mathrm{D} 1=$ first determination for individual

http://bigbotanicalgarden.org/individual/10224\#WXYZ= D2=second determination for individual

Note that when possible, several alternative terms from well-known vocabularies (i.e. Darwin Core, Dublin Core, XMP, FOAF) are used to describe the same property. All of these GUIDs are fictitious and should not be assumed to be resolvable. However, the actual RDF example files can be downloaded by appending the file name in the example to

http://bioimages.vanderbilt.edu/rdf/examples/, e.g.

http://bioimages.vanderbilt.edu/rdf/examples/25993.rdf

\section{Living botanical garden specimen (O2)}

Contents of file http://bigbotanicalgarden.org/collection/25993.rdf containing the metadata for a living specimen identified as http://bigbotanicalgarden.org/collection/25993

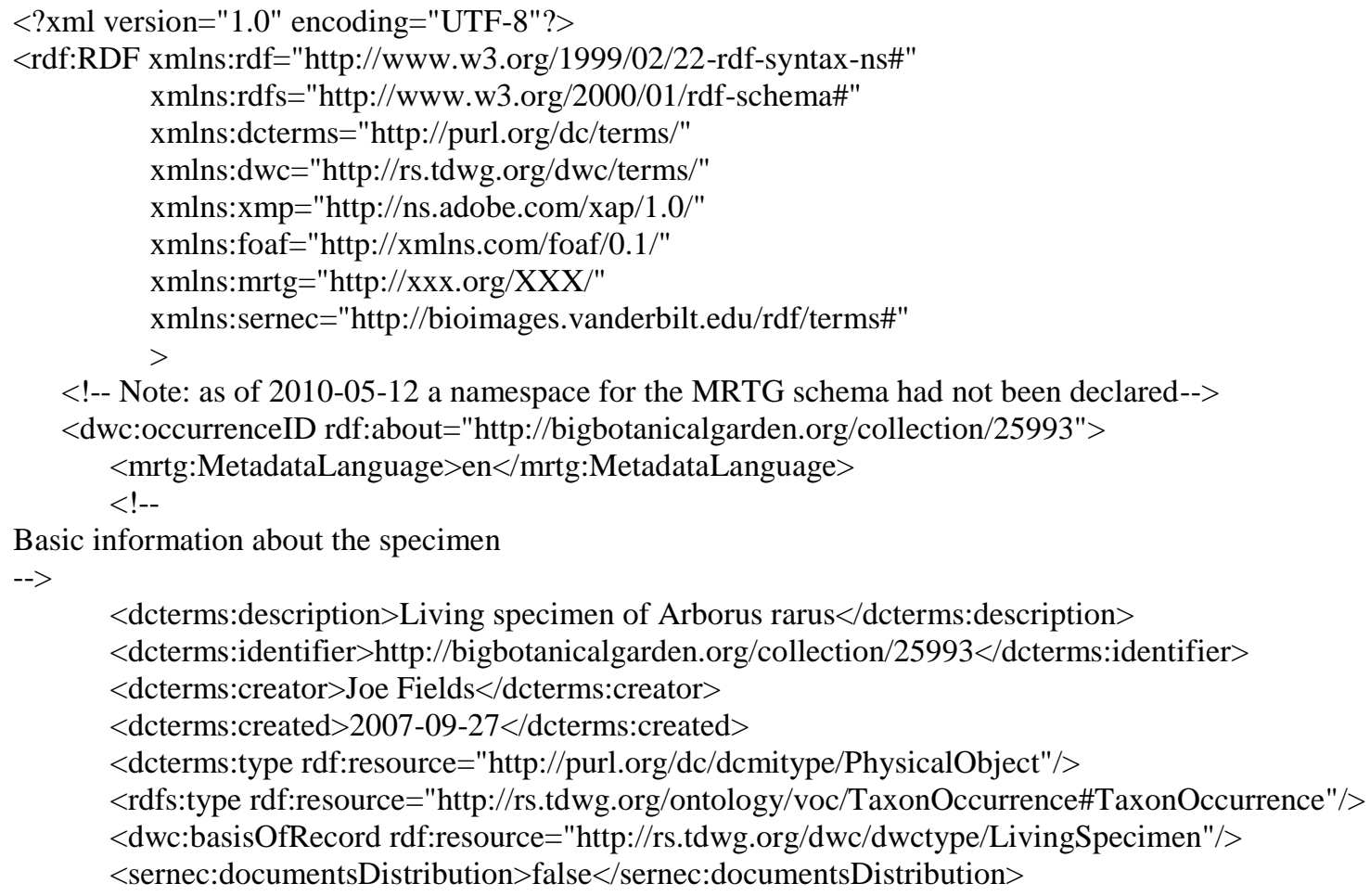




\section{BASKAUF - ORGANIZATION OF OCCURRENCE-RELATED BIODIVERSITY RESOURCES}

<dwc:collectionID rdf:resource="http://biocol.org/urn:lsid:biocol.org:col:34818"/> $<!--$

Relationships of the specimen to other resources

$-->$

<foaf:isPrimaryTopicOf rdf:resource="http://bigbotanicalgarden.org/collection/25993.rdf"/>

<foaf:isPrimaryTopicOf rdf:resource="http://bigbotanicalgarden.org/collection/25993.htm"/>

<sernec:derivedFrom rdf:resource="http://saveourspecies.org/germplasm/54899"/>

<dwc:individualID rdf:resource="http://bigbotanicalgarden.org/individual/10224"/>

<sernec:derivativeOccurrence rdf:resource="http://bigbotanicalgarden.org/image/52011"/>

<dwc:associatedMedia rdf:resource="http://bigbotanicalgarden.org/image/52011"/>

<foaf:depiction rdf:resource="http://bigbotanicalgarden.org/image/52011"/>

<sernec:derivativeOccurrence rdf:resource="http://www.ncbi.nlm.nih.gov/nuccore/BQ12345"/>

$<$ dwc:associatedSequences rdf:resource="http://www.ncbi.nlm.nih.gov/nuccore/BQ12345"/>

<sernec:usedInDetermination rdf:resource="http://bigbotanicalgarden.org/individual/10224\#WXYZ"/> $<!--$

Location information

$-->$

$\langle$ dwc:decimalLatitude $>41.09834</$ dwc:decimalLatitude $>$

$<$ dwc:decimalLongitude $>-121.17611</$ dwc:decimalLongitude $>$

$<$ dwc:geodeticDatum>EPSG:4326</dwc:geodeticDatum>

$<$ dwc:coordinateUncertaintyInMeters $>10</$ dwc:coordinateUncertaintyInMeters $>$

$<$ dwc:locality $>$ Big Botanical Garden $</$ dwc:locality $>$

$<$ !-- Note: the "false" value of sernec:documentsDistribution means that the creation of this

occurrence does not serve as evidence that a taxon representative exists in the wild at the

location given. (The location is the place where the LivingSpecimen was planted.) -->

$</$ dwc:occurrenceID $>$

$<!--$

Information about the metadata itself

$-->$

<rdf:Description rdf:about="http://bigbotanicalgarden.org/collection/25993.rdf">

<dcterms:description>RDF formatted description of the living specimen

http://bigbotanicalgarden.org/collection/25993</dcterms:description>

$<$ dcterms:creator $>$ Big Botanical Garden $</$ dcterms:creator $>$

$<$ dcterms:created $>2008-09-08 \mathrm{~T} 12: 01: 30-0800</$ dcterms:created $>$

$<$ dcterms:language $>$ en $</$ dcterms:language $>$

<dcterms:modified>2009-10-07T09:14:08-0800</dcterms:modified>

<xmp:MetadataDate> 2009-10-07T09:14:08-0800</xmp:MetadataDate>

<dcterms:references rdf:resource="http://bigbotanicalgarden.org/collection/25993"/>

$<$ foaf:primaryTopic rdf:resource="http://bigbotanicalgarden.org/collection/25993"/>

$</$ rdf:Description $>$

$</$ rdf:RDF $>$

\section{Still image (O4) of a living specimen}

Contents of file http://bigbotanicalgarden.org/image/52011.rdf containing the metadata for an image identified as urn:Isid:bigbotanicalgarden.org:image:52011 of a living specimen identified as http://bigbotanicalgarden.org/collection/25993

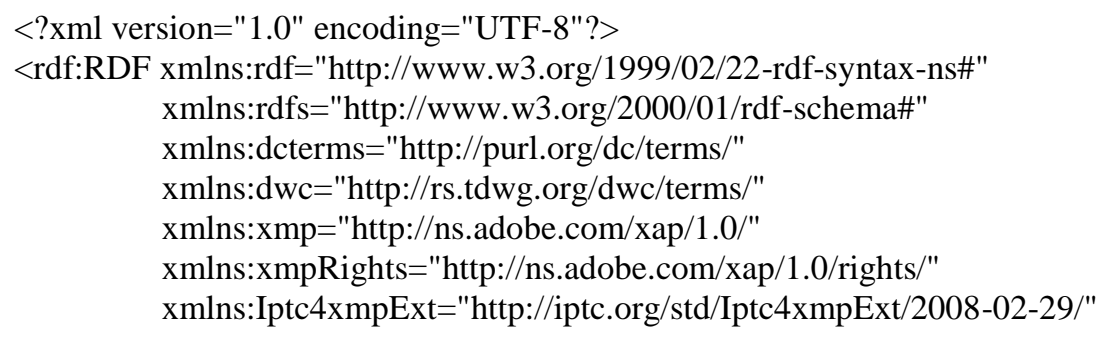




\title{
BASKAUF - ORGANIZATION OF OCCURRENCE-RELATED BIODIVERSITY RESOURCES
}

\author{
xmlns:foaf="http://xmlns.com/foaf/0.1/" \\ xmlns:mix="http://www.loc.gov/mix/v20" \\ xmlns:mrtg="http://xxx.org/XXX/" \\ xmlns:sernec="http://bioimages.vanderbilt.edu/rdf/terms\#" \\ <dwc:occurrenceID rdf:about="http://bigbotanicalgarden.org/image/52011"> \\ $<$ mrtg:MetadataLanguage > en </mrtg:MetadataLanguage> \\ $<!--$ \\ Basic information about the image \\ $-->$ \\ $<$ dcterms:description>Living specimen image with GUID \\ http://bigbotanicalgarden.org/image/52011</dcterms:description> \\ <dcterms:identifier>http://bigbotanicalgarden.org/image/52011</dcterms:identifier> \\ $<$ dcterms:creator $>$ Fred Fotografer $</$ dcterms:creator $>$ \\ <dcterms:created>2009-08-03T14:24:02-0800</dcterms:created> \\ <dcterms:type rdf:resource="http://purl.org/dc/dcmitype/StillImage"/> \\ <rdfs:type rdf:resource="http://rs.tdwg.org/ontology/voc/TaxonOccurrence\#TaxonOccurrence"/> \\ $<!--$ \\ DigitalStillImage does not have a normative URI because it is not (yet) an accepted DwC type \\ $-->$ \\ $<$ dwc:basisOfRecord $>$ DigitalStillImage $</$ dwc:basisOfRecord $>$ \\ $<$ sernec:documentsDistribution $>$ false $</$ sernec:documentsDistribution> \\ $<$ dwc:collectionID rdf:resource="http://biocol.org/urn:lsid:biocol.org:col:34818"/> \\ $<!--$ \\ specified \\ Other properties of the image related to intellectual property rights, use and attribution guidelines, etc. \\ by the TDWG/GBIF Multimedia Resource Task Group schema \\ $-->$ \\ <dcterms:rights>(c) 2009 Big Botanical Garden</dcterms:rights> \\ <xmpRights:owner>Big Botanical Garden</xmpRights:owner> \\ <Iptc4xmpExt:creditLine>Image by Fred Fotografer, Big Botanical Garden</Iptc4xmpExt:creditLine> \\ $<$ dwc:title>Living specimen of Arborus rarus in Big Botanical Garden</dwc:title> \\ <xmpRights:UsageTerms>Available under Creative Commons Attribution-Noncommercial-Share Alike 3.0 \\ license</xmpRights:UsageTerms > \\ $<$ xmpRights:WebStatement>http://creativecommons.org/licenses/by-nc- \\ sa/3.0/us/</xmpRights:WebStatement> \\ $<$ dcterms:description>Image of living Arborus rarus specimen (whole tree) $</$ dcterms:description> \\ <mrtg:attributionLinkURL>http://bigbotanicalgarden.org/about.htm</mrtg:attributionLinkURL> \\ <mrtg:attributionLogoURL>http://bigbotanicalgarden.org/logo.jpg</mrtg:attributionLogoURL> \\ $<!--$ \\ Relationships of the image to other resources \\ $-->$ \\ <foaf:isPrimaryTopicOf rdf:resource="http://bigbotanicalgarden.org/image/52011.rdf"/> \\ <foaf:isPrimaryTopicOf rdf:resource="http://bigbotanicalgarden.org/image/52011.htm"/> \\ <sernec:derivedFrom rdf:resource="http://bigbotanicalgarden.org/collection/25993"/> \\ <foaf:depicts rdf:resource="http://bigbotanicalgarden.org/collection/25993"/> \\ <dwc:individualID rdf:resource="http://bigbotanicalgarden.org/individual/10224"/> \\ $<!--$ \\ Location information \\ <dwc:decimalLatitude >41.09834</dwc:decimalLatitude> \\ $<$ dwc:decimalLongitude>-121.17611</dwc:decimalLongitude > \\ $<$ dwc:geodeticDatum>EPSG:4326</dwc:geodeticDatum> \\ $<$ dwc:coordinateUncertaintyInMeters $>10</$ dwc:coordinateUncertaintyInMeters $>$ \\ $\langle$ dwc:locality $>$ Big Botanical Garden</dwc:locality $>$ \\ $<$ !-- Note: this is the location where the image was created (i.e. in Big Botanical Garden).
}




\section{BASKAUF - ORGANIZATION OF OCCURRENCE-RELATED BIODIVERSITY RESOURCES}

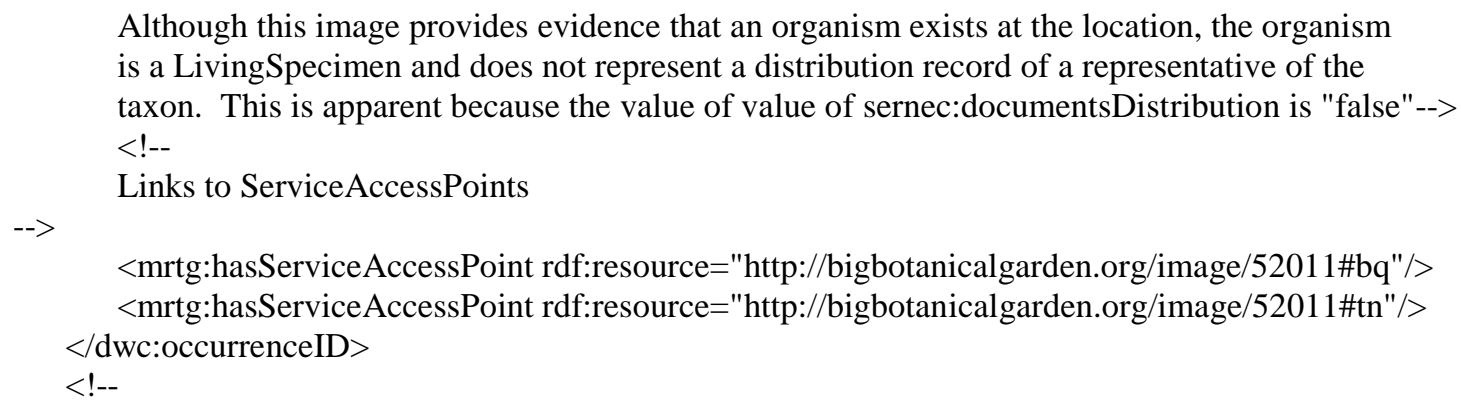

ServiceAccessPoints provide information about alternative versions of the image having different resolutions.

Note that the access URLs do not have to be related to the HTTP URI GUID of the image and can therefore be changed at will. The access URLs can also contain query strings if desired (but take care to escape the "\&" character).

\section{Still image of a living organism in the wild (e.g. live plant image in Fig. 6)}

Contents of file http://bigbotanicalgarden.org/image/44321.rdf containing the metadata for an image identified as http://bigbotanicalgarden.org/image/44321 of an individual in its environment identified as http://bigbotanicalgarden.org/individual/10224

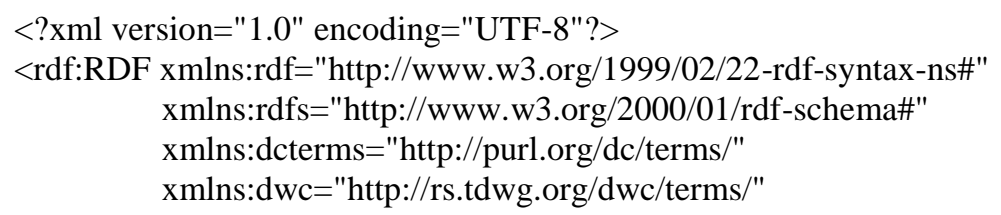




\title{
BASKAUF - ORGANIZATION OF OCCURRENCE-RELATED BIODIVERSITY RESOURCES
}

\author{
xmlns:xmp="http://ns.adobe.com/xap/1.0/" \\ xmlns:xmpRights="http://ns.adobe.com/xap/1.0/rights/" \\ xmlns:Iptc4xmpExt="http://iptc.org/std/Iptc4xmpExt/2008-02-29/" \\ xmlns:foaf="http://xmlns.com/foaf/0.1/" \\ xmlns:mix="http://www.loc.gov/mix/v20" \\ xmlns:mrtg="http://xxx.org/XXX/" \\ xmlns:sernec="http://bioimages.vanderbilt.edu/rdf/terms\#" \\ $>$ \\ <dwc:occurrenceID rdf:about="http://bigbotanicalgarden.org/image/44321"> \\ $<$ mrtg:MetadataLanguage > en </mrtg:MetadataLanguage > \\ $<!--$ \\ Basic information about the image \\ $-->$ \\ <dcterms:description>Image with GUID http://bigbotanicalgarden.org/image/44321 of individual in the \\ wild</dcterms:description> \\ <dcterms:identifier>http://bigbotanicalgarden.org/image/44321</dcterms:identifier> \\ $<$ dcterms:creator $>$ Frederico Fotografo</dcterms:creator $>$ \\ $<$ dcterms:created>1998-11-16T09:14:36-0300</dcterms:created> \\ <dcterms:type rdf:resource="http://purl.org/dc/dcmitype/StillImage"/> \\ <rdfs:type rdf:resource="http://rs.tdwg.org/ontology/voc/TaxonOccurrence\#TaxonOccurrence"/> \\ $<!--$ \\ $-->$ \\ DigitalStillImage does not have a normative URI because it is not (yet) an accepted DwC type \\ $<$ dwc:basisOfRecord $>$ DigitalStillImage </dwc:basisOfRecord $>$ \\ $<$ sernec:documentsDistribution>true $</$ sernec:documentsDistribution> \\ $<$ dwc:recordedBy $>$ Frederico Fotografo $</$ dwc:recordedBy $>$ \\ <dwc:eventDate>1998-11-16T09:14:36-0300</dwc:eventDate> \\ <dwc:collectionID rdf:resource="http://biocol.org/urn:lsid:biocol.org:col:34818"/> \\ $<!--$ \\ Other properties of the image related to intellectual property rights, use and attribution guidelines, etc. \\ specified by the TDWG/GBIF Multimedia Resource Task Group schema \\ $-->$ \\ <dcterms:rights>(c) 1998 Instituto de Plantas Nativas</dcterms:rights> \\ <xmpRights:owner>Instituto de Plantas Nativas</xmpRights:owner> \\ $<$ Iptc4xmpExt:creditLine>Image by Frederico Fotografo, Instituto de Plantas \\ Nativas</Iptc4xmpExt:creditLine> \\ $<$ dwc:title $>$ Arborus rarus in Rio Grande do Sul, Brasil</dwc:title $>$ \\ $<$ xmpRights:UsageTerms>Available under Creative Commons Attribution-Noncommercial-Share Alike 3.0 \\ license</xmpRights:UsageTerms $>$ \\ $<$ xmpRights:WebStatement>http://creativecommons.org/licenses/by-nc- \\ sa/3.0/br/</xmpRights:WebStatement > \\ $<$ dcterms:description>Image of living Arborus rarus specimen (whole tree)</dcterms:description> \\ <mrtg:attributionLinkURL>http://bigbotanicalgarden.org/about.htm</mrtg:attributionLinkURL> \\ <mrtg:attributionLogoURL>http://bigbotanicalgarden.org/logo.jpg</mrtg:attributionLogoURL> \\ $<$ !-- Relationships of the image to other resources. \\ $-->$ \\ <foaf:isPrimaryTopicOf rdf:resource="http://bigbotanicalgarden.org/image/44321.rdf"/> \\ <foaf:isPrimaryTopicOf rdf:resource="http://bigbotanicalgarden.org/image/44321.htm"/> \\ <sernec:derivedFrom rdf:resource="http://bigbotanicalgarden.org/individual/10224"/> \\ <foaf:depicts rdf:resource="http://bigbotanicalgarden.org/individual/10224"/> \\ <dwc:individualID rdf:resource="http://bigbotanicalgarden.org/individual/10224"/> \\ <sernec:usedInDetermination rdf:resource="http://bigbotanicalgarden.org/individual/10224\#ABCD"/> \\ $<!--$ \\ Location information \\ $-->$ \\ <dwc:decimalLatitude>-29.633905</dwc:decimalLatitude>
}




\title{
BASKAUF - ORGANIZATION OF OCCURRENCE-RELATED BIODIVERSITY RESOURCES
}

\author{
$<$ dwc:decimalLongitude $>-50.204086</$ dwc:decimalLongitude $>$ \\ $<$ dwc:geodeticDatum>EPSG:4326</dwc:geodeticDatum> \\ $<$ dwc:coordinateUncertaintyInMeters $>10</$ dwc:coordinateUncertaintyInMeters $>$ \\ <dwc:locality>east side of RS-484 $7.9 \mathrm{~km}$ north of junction with BR $101</$ dwc:locality $>$ \\ $<$ !-- Note: this is the location where the tree was found in the wild. A value for \\ sernec:documentsDistribution \\ of "true" indicates that this represents a distribution record of a representative of the taxon.--> \\ $<!--$ \\ Links to ServiceAccessPoints \\ $-->$ \\ <mrtg:hasServiceAccessPoint rdf:resource="http://bigbotanicalgarden.org/image/44321\#bq"/> \\ <mrtg:hasServiceAccessPoint rdf:resource="http://bigbotanicalgarden.org/image/44321\#tn"/> \\ $</$ dwc:occurrenceID $>$ \\ <mrtg:hasServiceAccessPoint rdf:about="http://bigbotanicalgarden.org/image/44321\#bq"> \\ $<$ mrtg:variant>Best Quality</mrtg:variant> \\ <mrtg:accessURL>http://bigbotanicalgarden.org/raw-imports/dsc00234.jpg</mrtg:accessURL> \\ $<$ dcterms:format>image/jpeg</dcterms:format> \\ $<$ mix:imageWidth>1440</mix:imageWidth> \\ $<$ mix:imageHeight $>2160</$ mix:imageHeight $>$ \\ $</$ mrtg:hasServiceAccessPoint $>$ \\ <mrtg:hasServiceAccessPoint rdf:about="http://bigbotanicalgarden.org/image/44321\#tn"> \\ $<$ mrtg:variant>Thumbnail</mrtg:variant> \\ <mrtg:accessURL>http://bigbotanicalgarden.org/thumbs/44321.jpg</mrtg:accessURL > \\ $<$ dcterms:format>image/jpeg</dcterms:format> \\ $<$ mix:imageWidth $>67</$ mix:imageWidth> \\ $<$ mix:imageHeight $>100</$ mix:imageHeight $>$ \\ $</$ mrtg:hasServiceAccessPoint $>$ \\ $<!--$ \\ Information about the metadata itself \\ $-->$ \\ <rdf:Description rdf:about="http://bigbotanicalgarden.org/image/44321.rdf"> \\ $<$ dcterms:description $>$ RDF formatted description of the live organism image \\ http://bigbotanicalgarden.org/image/44321</dcterms:description> \\ $<$ dcterms:creator $>$ Big Botanical Garden</dcterms:creator $>$ \\ <dcterms:created>1999-03-15T14:54:51-0800</dcterms:created> \\ $<$ dcterms:language $>$ en $</$ dcterms:language $>$ \\ <dcterms:modified>2009-10-07T09:14:08-0800</dcterms:modified> \\ <xmp:MetadataDate>2009-10-07T09:14:08-0800</xmp:MetadataDate> \\ <dcterms:references rdf:resource="http://bigbotanicalgarden.org/image/44321"/> \\ <foaf:primaryTopic rdf:resource="http://bigbotanicalgarden.org/image/44321"/> \\ $</$ rdf:Description $>$ \\ $</$ rdf:RDF $>$
}

For a functioning example of an occurrence GUID, enter

http://bioimages.vanderbilt.edu/baskauf/66921 into a web or rdf browser. Through content negotiation, it will resolve to either http://bioimages.vanderbilt.edu/baskauf/66921.htm if content-type text/html is requested by a web browser, or http://bioimages.vanderbilt.edu/baskauf/66921.rdf if content-type application/rdf $+x m l$ is requested by an RDF browser. 
Appendix B. Sample RDF file for the resource representing an individual in Fig. 10 and the determination resource in Fig. 11 using the example of the tree (I) and determination 2 (D2) in the botanical garden scheme (Figs. 6 and 8). The same GUIDs were used as in Appendix A.

Contents of file http://bigbotanicalgarden.org/individual/10224.rdf containing the metadata for an individual organism identified as http://bigbotanicalgarden.org/individual/10224 and its determinations

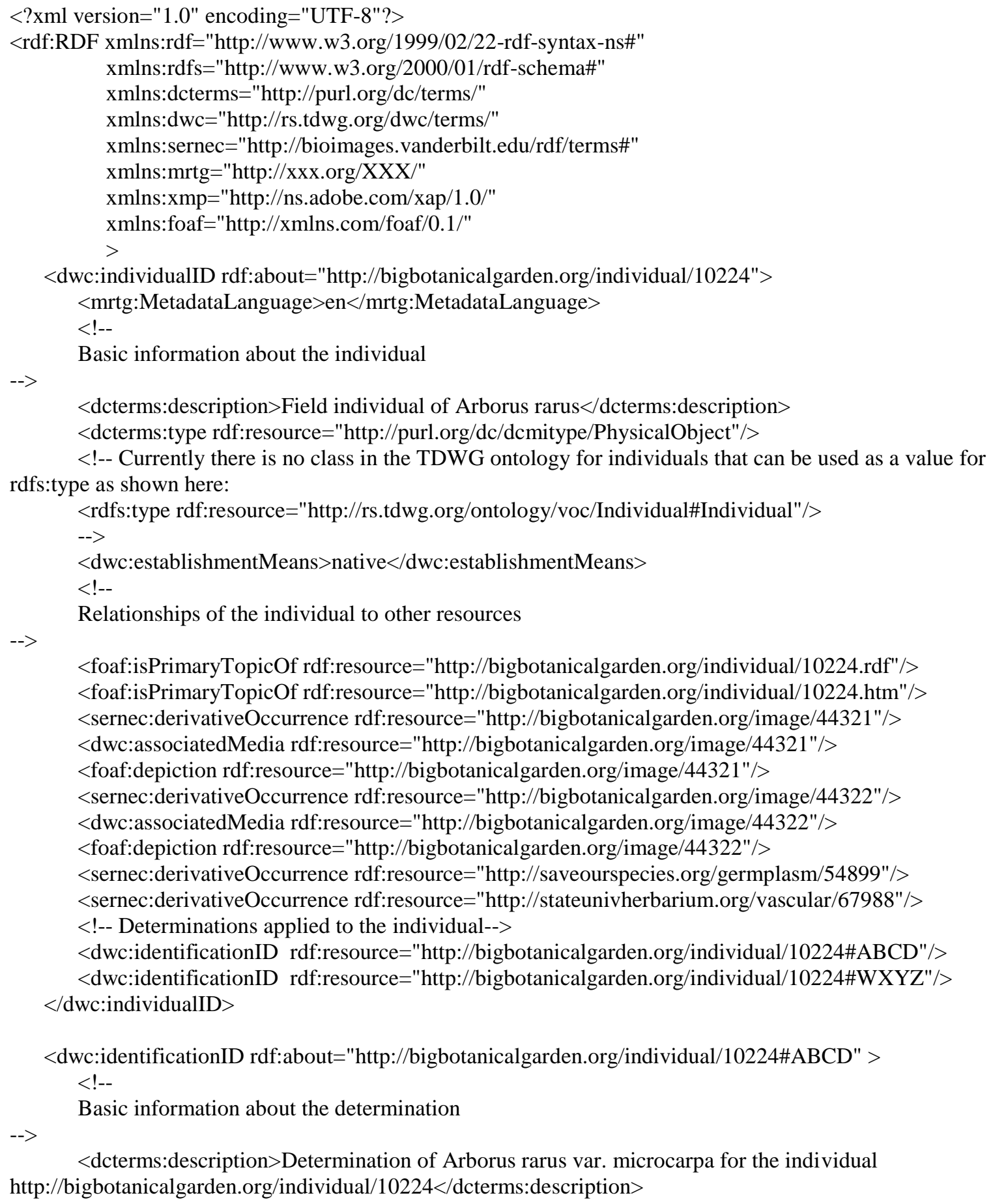




\section{BASKAUF - ORGANIZATION OF OCCURRENCE-RELATED BIODIVERSITY RESOURCES}

<rdfs:type rdf:resource ="http://rs.tdwg.org/ontology/voc/TaxonOccurrence\#Identification" / > $\langle$ dwc:identifiedBy $>$ Juanita Naturalista $</ d w c:$ identifiedBy $>$

$<$ dwc:dateIdentified $>1998-11-17</$ dwc:dateIdentified $>$

$<!--$

Relationship of the determination to other resources

$-->$

<sernec:identifiesIndividual rdf:resource="http://bigbotanicalgarden.org/individual/10224"/>

$<$ !-- This determination was based on the two images of the source individual in the wild: -->

<sernec:basedOnOccurrence rdf:resource="http://bigbotanicalgarden.org/image/44321"/>

<sernec:basedOnOccurrence rdf:resource="http://bigbotanicalgarden.org/image/44322"/>

$<$ !-- and was also based on examination of a seed collected from the tree: -->

<sernec:basedOnOccurrence rdf:resource="http://saveourspecies.org/germplasm/54899"/>

$<$ dwc:taxonConceptID rdf:resource="http://lod.geospecies.org/ses/ABCD"/>

$<!--$

Direct literals for the determination can be found here without resolving the taxonConceptID

$-->$

$\langle$ dwc:genus $>$ Arborus $</$ dwc:genus $>$

$<$ dwc:specificEpithet $>$ rarus $</$ dwc:specificEpithet $>$

$<$ dwc:infraspecificEpithet $>$ microcarpa $</$ dwc:infraspecificEpithet $>$

$<$ dwc:taxonRank $>$ variety $</$ dwc:taxonRank $>$

$<$ dwc:scientificNameAuthorship $>$ J. Rodriguez $</$ dwc:scientificNameAuthorship $>$

$</$ dwc:identificationID $>$

<dwc:identificationID rdf:about="http://bigbotanicalgarden.org/individual/10224\#WXYZ" > $<$ dcterms:description $>$ Determination of Arborus rarus for the individual

http://bigbotanicalgarden.org/individual/10224</dcterms:description>

<rdfs:type rdf:resource ="http://rs.tdwg.org/ontology/voc/TaxonOccurrence\#Identification" / >

$\langle$ dwc:identifiedBy $>$ Jane Jardin $</$ dwc:identifiedBy $>$

$<$ dwc:dateIdentified $>2008-06-24</$ dwc:dateIdentified $>$

$<!--$

$->$

Relationship of the determination to other resources

<sernec:identifiesIndividual rdf:resource="http://bigbotanicalgarden.org/individual/10224"/>

$<$ !-- This determination was based on the botanical garden LivingSpecimen: -->

<sernec:basedOnOccurrence rdf:resource="http://bigbotanicalgarden.org/collection/25993"/>

$<!--$ and was also based on the image of a specimen in an herbarium: -->

<sernec:basedOnOccurrence rdf:resource="http://stateunivherbarium.org/vascular/67988\#img"/>

$<$ dwc:taxonConceptID rdf:resource="http://lod.geospecies.org/ses/WXYZ"/>

$<!--$

Direct literals for the determination can be found here without resolving the taxonConceptID

$-->$

$\langle$ dwc:genus $>$ Arborus $</$ dwc:genus $>$

$<$ dwc:specificEpithet $>$ rarus $</$ dwc:specificEpithet $>$

$<$ taxonRank $>$ species $</$ taxonRank $>$

$<$ dwc:scientificNameAuthorship $>$ L. $</ d w c: s c i e n t i f i c N a m e A u t h o r s h i p>$

$</$ dwc:identificationID $>$

$<!--$

Information about the metadata itself

$->$

<rdf:Description rdf:about="http://bigbotanicalgarden.org/individual/10224.rdf" >

$<$ dcterms:description $>$ RDF formatted description of the field individual

http://bigbotanicalgarden.org/individual/10224</dcterms:description>

$<$ dcterms:creator $>$ Big Botanical Garden $</$ dcterms:creator $>$

$<$ dcterms:created >1999-03-15T14:54:54-0800</dcterms:created>

$<$ dcterms:language $>$ en $</$ dcterms:language $>$ 
<dcterms:modified>2009-10-07T09:14:08-0600</dcterms:modified> <xmp:MetadataDate>2009-10-07T09:14:08-0600</xmp:MetadataDate> <dcterms:references rdf:resource="http://bigbotanicalgarden.org/individual/10224"/> <foaf:primaryTopic rdf:resource="http://bigbotanicalgarden.org/individual/10224"/> $</$ rdf:Description $>$ $</$ rdf:RDF $>$

For a functioning example of a GUID for an individual organism, enter http://bioimages.vanderbilt.edu/ind-baskauf/66920 into a web or rdf browser. Through content negotiation, it will resolve to either http://bioimages.vanderbilt.edu/ind-baskauf/66920.htm if content-type text/html is requested by a web browser, or http://bioimages.vanderbilt.edu/baskauf/66921.rdf if content-type application/rdftxml is requested by an RDF browser. 